Cattaleeya Pattamaprom Ronald G. Larson

\section{Predicting the linear viscoelastic properties of monodisperse and polydisperse polystyrenes and polyethylenes}

Received: 11 October 2000

Accepted: 17 May 2001
C. Pattamaprom

Department of Chemical Engineering

Thammasat University, Pratumthani 12121

Thailand

R. G. Larson $(\bowtie)$

Department of Chemical Engineering

University of Michigan

Ann Arbor, MI 48109, USA

e-mail: rlarson@engin.umich.edu

\begin{abstract}
For linear homopolymers the linear viscoelastic predictions of the double reptation model are compared to those of a recent, more detailed model, the "dual constraint model" and to experimental data for monodisperse, bidisperse, and polydisperse polystyrene melts from several laboratories. A mapping procedure is developed that links the empirical parameter $\mathrm{K}$ of the double reptation model to the molecular parameter $\tau_{\mathrm{e}}$ of the dual constraint model, thereby allowing the parameter $\mathrm{K}$ to be related to molecular characteristics such as the monomeric friction coefficient $\zeta$. Once $\mathrm{K}$ (or $\tau_{\mathrm{e}}$ ) are determined from data for monodisperse polymers, the double reptation model predicts that for fixed weight-average molecular weight $\mathrm{M}_{\mathrm{w}}$, the zero-shear viscosity $\eta_{0}$ increases slightly with increasing
\end{abstract}

polydispersity $\mathrm{M}_{\mathrm{w}} / \mathrm{M}_{\mathrm{n}}$ for $\log$ normal distributions, while for the dual constraint model $\eta_{0}$ is almost independent of $M_{w} / M_{n}$. Experimental data for polystyrenes show no increase (or even a slight decrease) in $\eta_{0}$ with increasing $M_{w} / M_{n}$ at fixed $\mathrm{M}_{\mathrm{w}}$, indicating a deficiency in the double reptation model. The dual constraint theory is also applied to hydrogenated polybutadienes and commercial high-density polyethylenes, where we believe it can be used to indicate the presence of long side branches, which are difficult to detect by other analytic methods.

Key words Dual constraint model · Double reptation model .

Polydispersity · Polystyrene .

Polyethylene $\cdot$ Hydrogenated polybutadiene

\section{Introduction}

Recently there has been a high level of interest in relating quantitatively the linear viscoelasticity of polymer melts to their molecular weight distributions (Montfort et al. 1979, 1986; Wasserman and Graessley 1992; Mead 1994; Léonardi et al. 1998). Interest in this topic has been stimulated by the prospect of inferring the molecular weight distribution (MWD) of a melt from its linear viscoelasticity alone (without the need for expensive GPC analysis), and by the development of the "double reptation" formula (Tuminello 1986; Tsenoglou 1987, 1991; des Cloizeau 1988; Milner 1996) which provides a single formula relating the storage and loss moduli $\left(\mathrm{G}^{\prime}\right.$ and $G^{\prime \prime}$ ) to the MWD. While reasonable success has been obtained in extracting MWD from $G^{\prime}$ and $G^{\prime \prime}$ using double reptation and related theories, systematic studies have not yet been carried out to determine the range of weight-averaged molecular weights $\mathrm{M}_{\mathrm{w}}$ and the range of polydispersities over which the double reptation theory might be trusted to give accurate results.

In addition, theories that are potentially more accurate than double reptation have recently been proposed that have not systematically been compared with double reptation and with experimental data to determine their relative accuracies. For example, while it is sometimes assumed that, at fixed $\mathrm{M}_{\mathrm{w}}$, the zeroshear viscosity $\eta_{0}$ is independent of the ratio of 
weight-to-number averaged molecular weight $M_{w} / M_{n}$. This assumption seems not to have been systematically tested, nor is it known whether the experimental dependence of $\eta_{0}$ on $\mathrm{M}_{\mathrm{w}} / \mathrm{M}_{\mathrm{n}}$ is predicted correctly by double reptation or other theories.

Here we will investigate these issues by assembling literature data for the linear viscoelasticity of wellcharacterized monodisperse, bidisperse, and polydisperse polystyrene melts. We will compare these data to predictions of two promising theories, namely the "double reptation" theory and the recent "dual constraint" theory of Mead et al. (1997). We select polystyrene as our model melt primarily because of the wealth of available literature data, and because we can be confident that polystyrene melts lack any long side branches. It is well known that even a very low level of long side branching can have a dramatic effect on linear viscoelastic properties (Carella et al. 1986; Janzen and Colby 1999). We also make more limited comparisons of the dual constraint model to data of well-characterized hydrogenated polybutadienes, which are chemically equivalent to polyethylenes.

The double reptation model is based on the original Doi-Edward model plus a reptative constraint-release mechanism. The predictions of this model in monodisperse polymers are good in the terminal regime (i.e., low frequency regime) but very poor in the intermediateand high-frequency regime. In polydisperse systems, the predictions of the double reptation model in the intermediate frequency regime are dominated by the terminal relaxation of chains shorter than the longest ones. The model therefore appears to give good predictions over a wider frequency than it does for monodisperse melts. As a consequence, the double reptation model is considered an acceptable model for predicting linear viscoelastic rheological properties of monodisperse entangled polymers in the terminal relaxation regime and for polydisperse entangled polymers even outside this regime. Note, however, that the model is expected to break down in the limit of long chains in a matrix of short ones, where the long chain must then relax via the constraint release Rouse mechanism, not captured in the double reptation scheme.

One would like to extend the double reptation model so that it gives more accurate predictions over a wider range of frequencies. One would also like a model that can apply to monodisperse and polydisperse branched polymers, such as stars, as well as to linear molecules. To accomplish these objectives, one must incorporate contour-length fluctuations into the model. In addition, it is well known that for star polymers, constraint release has a more dramatic effect than can be accounted for by a simple generalization of the "double reptation" idea. This effect, called "dynamic dilution" or "tube dilation," is a widening of the tube at long times after a step strain due to the rapid relaxation of some of the constraints that define the tube. Marrucci (1985) and Ball and McLeish (1989) developed self-consistent calculations to compute simultaneously both the instantaneous relaxation function and the instantaneous tube diameter as functions of time after a step strain. Marrucci's calculation was developed for linear polymers, while that of Ball and McLeish was developed for star polymers. The method of Ball and McLeish has proven to be extremely accurate in the prediction of the relaxation spectrum of monodisperse star polymers. Still, a general theory that can predict the relaxation behavior of polydisperse linear and star branched polymers is needed.

Recently, Mead et al. (1997) developed the "dual constraint" model, which generalizes the double reptation model by incorporating contour-length fluctuations and constraint release by double reptation and tube dilation, all in a single, relatively simple, algorithm. This is accomplished through the artifice of a "two-tube" calculation, wherein the relaxation of a chain (linear or star) in a fixed matrix is undertaken first. This calculation is then integrated to yield a single function $\phi^{*}(t)$, which is the fraction of tube segments that are unrelaxed at a time $t$ after the step strain. This "dilution" function is then incorporated into a second calculation of the relaxation, which is the same as that in a fixed tube, except that the function $\phi^{*}(t)$, calculated in the fixed tube, is used to dilute the density of entanglements used in the calculation of the fluctuation potential. This approach, while not self-consistent in the way the BallMcLeish calculation is, nevertheless captures much of the "tube dilation" effect. This is demonstrated in the Appendix, where it is shown that for monodisperse star polymers, the "dual constraint" model yields an acceleration of relaxation similar to that produced by the conventional Ball-McLeish argument.

The work of Pattamaprom et al. (2000) enhanced that of Mead et al. (1997) by devising an accurate "cross-over" function for early-to-late time fluctuations; this cross-over function is presented again in what follows. In addition, the work of Pattamaprom et al. included constraint release Rouse motion, which was absent from the work of Mead et al. Pattamaprom et al. (2000) showed that this revised "dual constraint" model produces remarkably accurate predictions for monodisperse and bidisperse linear and star polymer melts, even without adjustable parameters. A single, successful, comparison was also made with broadly polydisperse polystyrene at $150{ }^{\circ} \mathrm{C}$ (Wasserman and Graessley 1992) with one adjustable parameter (due to the lack of a reliable literature value of the monomeric friction coefficient at $150^{\circ} \mathrm{C}$ ).

Since both the double reptation and dual constraints models work equally well in the terminal regime, the two models can be used interchangeably in this regime and their parameters can be found and correlated with each other for monodisperse linear polymers. Here we 
provide a mapping scheme to retrieve the model parameter $\mathrm{K}$ of the double reptation model from the model parameter $\tau_{\mathrm{e}}$ of the dual-constraint model and vice versa. This correlation could be useful especially for high molecular weight polymers, where the terminal regime is often difficult to obtain, thus making the model parameter obtained from the terminal regime inaccurate. Since the dual constraint model is capable of intermediate-regime prediction, the mapping between the parameters $\tau_{\mathrm{e}}$ and $\mathrm{K}$ would give a more accurate $\mathrm{K}$ value and help alleviate the experimental difficulty in trying to reach the terminal regime either from low sensitivity at low-frequency measurements or from erroneous time-temperature superposition.

We also investigate here the effects of broad, continuous, molecular-weight distributions more thoroughly. We compare the predictions of the dualconstraint and double-reptation models with experimental linear viscoelastic data for polystyrenes at different values of $\mathrm{M}_{\mathrm{w}} / \mathrm{M}_{\mathrm{n}}$. We thereby find discrepancies between the experimental data and the predictions of the models, especially the double reptation model, which should motivate a search for improvement of the double reptation model to account more accurately for the effects of polydispersity.

Next, we apply the dual constraint model to predict the viscoelastic properties of linear and star hydrogenated polybutadienes (h-PBD) (Raju et al. 1979), which are the model polymers for linear and star-branched polyethylenes, respectively. For linear h-PBD, we will show that the zero-shear viscosities and linear viscoelastic moduli agree well with experimental data for the whole range of molecular weight studied. For star h-PBD, however, the predictions of linear viscoelastic moduli $\left(G^{\prime}\right.$ and $\left.G^{\prime \prime}\right)$ agree with the experimental data only when the activation potential of fluctuation and the plateau modulus are independently adjusted. The success of the dual constraint prediction for polydisperse linear polymers allows us to be able to differentiate the effect of polydispersity from that of long-chain branching in polyethylenes.

\section{Theoretical background}

The double reptation model and the dual constraint model will be utilized in evaluating the effect of polydispersity on the rheological properties of linear polymers.

The dual constraint model accounts for reptation, contour-length fluctuation, and constraint release by dynamic tube dilation and reptative constraint release. The double reptation model is like the dual constraint model except that it ignores dynamic tube dilation and accounts for contour-length fluctuations only indirectly by using an empirical 3.4 power-law dependence of relaxation time on molecular weight.
The following is a brief summary of the theory and model parameters of each model.

\section{The dual constraint model}

The dual constraint model combines two forms of constraint release in the dynamics of entangled polymers. The first form of constraint release is "double diffusion" which augments "double reptation" by inclusion of primitive path fluctuation as a mechanism of constraint release. The second form of constraint release is "dynamic dilution," which was introduced by Ball and McLeish (1989) to describe the time-dependent loosening of the effective entanglement network, or widening of the tube. The upper limit of the combined constraint release rate from both mechanisms is controlled by Rouse-like motions of the tube containing the test chain; these motions are called "constraint-release Rouse" processes (Viovy et al. 1991; Milner et al. 1998). Further discussion and description of the model are provided by Pattamaprom et al. (2000).

The diffusion equation for the tube survival probability $p_{\mathrm{i}}$ of chain type $i$ including constraint release is rewritten as

$\frac{\partial p_{i}\left(s_{i}, t\right)}{\partial t}=\frac{D_{i}}{L_{i}^{2}} \frac{\partial^{2} p_{i}\left(s_{i}, t\right)}{\partial s_{i}^{2}}-\frac{p_{i}\left(s_{i}, t\right)}{\tau_{\xi, i}\left(s_{i}\right)}$

where $p_{i}\left(s_{i}, t\right)$ is the survival probability of a tube segment occupied by a chain of type $i$ as a function of time $t$ and contour distance $s_{\mathrm{i}}$, where $s_{\mathrm{i}}$ ranges from 0 at the center of a polymer chain to $1 / 2$ at the chain end of a linear, or to 1 at the chain end of a star. $D_{\mathrm{i}}$, the curvilinear diffusion coefficient, is $\frac{L_{i}^{2}}{\tau_{d, i} \pi^{2}}$, where $L_{\mathrm{i}}$ is the average contour length of the tube and $\tau_{\mathrm{d}, \mathrm{i}}$ is the reptation time constant (Doi and Edwards 1986). $\tau_{\xi, \mathrm{i}}$ is the time constant for contourlength fluctuations in the presence of constraint release which, at early time and late time, can be expressed by interpolating between two formulas for early- and latetime fluctuations (Pattamaprom et al. 2000):

$\tau_{\text {early }, i}\left(s_{i}\right)=\frac{225}{256} \pi^{3} \frac{\tau_{R, i}}{c_{i}^{2}}\left(1-c_{i} s_{i}\right)^{4}\left(\frac{N_{e n, i}}{c_{i}}\right)^{2}$ and
$\tau_{\text {late }, i}\left(s_{i}, t\right)=\frac{\tau_{R, i}}{c_{i}^{2}} \exp \left(U_{\text {eff }}\left(s_{i}, t\right)\right)$

Here $c_{\mathrm{i}}$ is a prefactor, which equals 2 for linear polymers (since a linear polymer has two fluctuating chain ends), and is unity for star arms. $\mathrm{N}_{\mathrm{en}, \mathrm{i}}$ is the number of entanglements in chain $i$, which equals $\frac{M_{w, i}}{M_{e}}$. $\mathbf{M}_{\mathrm{w}, \mathrm{i}}$ is the weight-average molecular weight of chain $i$, and $\mathrm{M}_{\mathrm{e}}$ is the molecular weight between entanglements. $\tau_{\mathrm{R}, \mathrm{i}}$ is the longest Rouse stress relaxation time of chain $i$ given by $\tau_{R, i}=\frac{\zeta N_{i}^{2} b_{i}^{2}}{6 \pi^{2} k_{b} T}$, which is smaller than the rotational relaxation time $\tau_{\mathrm{r}}$ from Doi and Edwards by a factor of two (Doi and Edwards 1986, p.115). Thus, 
$\tau_{\mathrm{R}, \mathrm{i}}$ is related to $\tau_{\mathrm{d}, \mathrm{i}}$ by $\tau_{\mathrm{R}, \mathrm{i}}=\tau_{\mathrm{d}, \mathrm{i}} /\left(6 \mathrm{~N}_{\mathrm{en}, \mathrm{i}}\right)$. The formula used to interpolate between $\tau_{\text {early,i }}$ and $\tau_{\text {late, } i}$ in the transition zone is the geometric average of the two. Therefore, the expression for $\tau_{\xi, \mathrm{i}}$ at positions in the transition zone along the chain is given by

$\tau_{\xi, i}=\tau_{\text {early }, i} \quad$ when $\left(1-c_{i} s_{i}\right)<C_{1, i}$

$\tau_{\xi, i}=\sqrt{\tau_{\text {early }, i} \cdot \tau_{\text {late }, i}}$ when $C_{1, i}<\left(1-c_{i} s_{i}\right)<C_{2, i}$

$\tau_{\xi, i}=\tau_{\text {late }, i}$ when $\left(1-c_{i} s_{i}\right)>C_{2, i}$

where $\mathrm{C}_{1, \mathrm{i}}$ is the first crossover position of $\tau_{\text {early,i }}$ and $\tau_{\text {late, } \mathrm{i}}$ close to the chain end, and $\mathrm{C}_{2, \mathrm{i}}$ is the second crossover point deeper inside the tube. This expression is reproduced from Eq. (12) of Pattamaprom et al. (2000). (Note that the variable $c_{i}$ was incorrectly left out from that equation, as well as from Eq. (4) of Pattamaprom et al. 2000). The variable $c$ there is equivalent to $c_{i}$ in this paper, indicating the dependence of $c$ on the chemical species.)

$\mathrm{U}_{\text {eff }}\left(\mathrm{s}_{\mathrm{i}}, \mathrm{t}\right)$ is the reduced activation energy obtained by

$U_{e f f}\left(s_{i}, t\right)=\frac{15}{8} \frac{N_{e n, i}}{c_{i}} \phi^{*}(t)\left(1-c_{i} s_{i}\right)^{2}$

where $\phi^{*}(\mathrm{t})$ is the dilution factor calculated from the tube survival probability in a fixed matrix as described in Pattamaprom et al. (2000).

The overall survival probability $\phi_{\mathrm{i}}(\mathrm{t})$ of a tube occupied by chain $i$ can then be calculated from $p_{i}\left(s_{i}, t\right)$ by

$\phi_{i}(t)=\int_{0}^{1} p_{i}\left(s_{i}, t\right) d s_{i}$

and the average survival probability $\phi(\mathrm{t})$ of all tubes is

$\phi(t)=\sum w_{i} \phi_{i}(t)$

where $\mathrm{w}_{\mathrm{i}}$ is the weight fraction of chains of type $i$.

Local constraint-release events are described in terms of the double-diffusion mechanism, developed as an extension of double reptation (Tuminello 1986; Tsenoglou 1987, 1991; des Cloizeaux 1988) by Mead et al. (1997). This theory considers the survival probability of a binary topological interaction of a test chain with a surrounding chain. This mechanism accounts for the effect of the surrounding chains on the overall relaxation process by multiplying the average survival probability of the test chain by that of the constraints surrounding the chain. Without the constraint-release Rouse process, the multiplication would be $\phi^{2}(\mathrm{t})$. However, after including constraint-release Rouse processes (Viovy et al. 1991; Milner et al. 1998), the fraction of constraints $\phi^{\prime}(\mathrm{t})$ that block motion of a test chain at time $t$ can differ from the fraction of tube segments $\phi(t)$ that are still occupied at time $t$. Thus

$$
\begin{aligned}
& \phi^{\prime}(t)=\phi(t) \text { when } \phi(t)>\phi_{R}(t) \\
& \phi^{\prime}(t)=\phi_{R}(t) \text { when } \phi(t)<\phi_{R}(t)
\end{aligned}
$$

where $\phi_{R}(t)=\phi\left(t_{0}\right)\left(\frac{t}{t_{0}}\right)^{-1 / 2}$. (A detailed explanation of the constraint-release Rouse process has been given in Viovy et al. (1991) and Milner (1996). Here, we will merely adopt the formula from Milner et al. (1998) that constraint-release Rouse relaxation permits relaxation no faster with time than $\mathrm{t}^{-1 / 2}$.) $\mathrm{t}_{0}$ is the time when $\phi(\mathrm{t})$ begins to drop faster than $\mathrm{t}^{-1 / 2}$, and $\phi\left(\mathrm{t}_{0}\right)$ is the average survival probability at $t_{0}$. At time near zero, $\phi^{\prime}(\mathrm{t})$ equals $\phi(\mathrm{t})$. After that, at each time $t, \phi^{\prime}(\mathrm{t})$ is assigned the greater of the values of $\phi(t)$ and $\phi_{R}(t)$.

Constraint-release Rouse processes are introduced so that when relaxation is relatively rapid, i.e., faster than the square root of time, relaxation due to constraint release is controlled by the rate at which the test chain can explore the widening tube. This typically happens when chains undergo terminal relaxation due to reptation, since terminal relaxation is exponentially fast. However, it can also occur during rapid early-time fluctuations. Hence, to cover all cases, we introduce constraint release Rouse relaxation whenever the function $\phi^{\prime}(\mathrm{t})$ relaxes too rapidly for the chain to explore fully the widening tube, that is, whenever the relaxation rate is faster than the square root of time.

Thus, the overall survival probability of the binary interactions between the test chains and the surrounding chains becomes

$\Phi(t)=\phi(t) \phi^{\prime}(t)$

The relaxation modulus $\mathrm{G}(\mathrm{t})$ from the contributions of reptation, contour-length fluctuations, and constraint release can be obtained by multiplying $\Phi(\mathrm{t})$ by the plateau modulus $G_{N}^{0}$ :

$G(t)=G_{N}^{0} \Phi(t)=G_{N}^{0} \phi(t) \phi^{\prime}(t)$

The high-frequency Rouse processes, which are distinct from the low-frequency "constraint-release Rouse" processes, are incorporated via the Rouse relaxation modulus $G_{R, i}(t)$ using an approximate "fragmented Rouse" spectrum proposed by Milner and McLeish (1998):

$G_{R, i}(t)=\left[\frac{1}{3} G_{N}^{0} \sum_{k=1}^{N_{e n, i}} \exp \left(\frac{-k^{2} t}{\tau_{R, i}}\right)+G_{N}^{0} \sum_{k=N_{e n, i}}^{N_{i}} \exp \left(\frac{-k^{2} t}{\tau_{R, i}}\right)\right] \frac{1}{N_{e n, i}}$

(It should be noted that this same equation that appears in Pattamaprom et al. (2000) mistakenly left out the factor $\frac{1}{N_{e n . i}}$.)

Finally, the total stress-relaxation modulus $G_{\text {total }}$ combines $G(t)$ from Eq. (9) with the contribution from high-frequency Rouse processes (Eq. 10) giving

$G_{\text {total }}(t)=G(t)+\sum_{i} w_{i} G_{R, i}(t)$

The input parameters for the model are the same as those required for the original Doi-Edward model: the 
plateau modulus $G_{N}^{0}$, and the Rouse relaxation time of a segment of a chain $\tau_{\mathrm{e}} . \tau_{\mathrm{e}}$ is theoretically related to molecular characteristics of each polymer by $\tau_{\mathrm{e}}=\frac{\zeta a^{2} M_{e}}{3 \pi^{2} k_{p} T M_{0}}$, where $\zeta$ is the monomeric friction coefficient, $a$ is the tube diameter, $\mathrm{M}_{\mathrm{e}}$ is the molecular weight per entanglement segment, and $\mathrm{M}_{0}$ is the molecular weight of monomer. $\mathrm{k}_{\mathrm{B}}$ is the Boltzmann constant and $\mathrm{T}$ is the absolute temperature.

Our expression for $\tau_{e}$ contains one power of the entanglement molecular weight $\mathbf{M}_{\mathrm{e}}$ explicitly, and another power of $\mathbf{M}_{\mathrm{e}}$ implicitly, through the linear dependence of $\mathrm{a}^{2}$ on $\mathrm{M}_{\mathrm{e}}$. Since it is only the Rouse time, and not $\tau_{e}$ that appears in the original equations, the definition of $\tau_{e}$ is just a convenience, and does not affect the final equations. There are some real differences, however, between our expressions for $\tau_{\text {late }}$ and $\tau_{\text {early }}$ and those of Milner and McLeish (1997). One difference is that Milner and McLeish used a first-passage-time derivation of the pre-factor for $\tau_{\text {late }}$, which depends on the form of the potential, which, in their calculations, is known analytically. In our approach, the potential in dilating tube is derived numerically, and so there is no first-passage solution. Therefore, we have elected to use as a prefactor in both $\tau_{\text {late }}$ and $\tau_{\text {early }}$ simply the Rouse relaxation time for the stress, which is one-half the Rouse "rotational" relaxation time, as discussed in Pattamaprom et al. (2000) and in Doi and Edwards (1986, p. 115). These choices are not rigorous ones, but, as shown in Pattamaprom et al. (2000), they yield very good predictions for a wide variety of linear and star polymers. There is also the factor of $c_{i}$ that appears in Eq. (2), which is unity for star arms, and has the value 2 for linear chains. This is because linear chains can experience fluctuations from both ends. Please note also the inconsistency in the values of the entanglement spacings $M_{e}$ used in Milner and McLeish (1997). In their theoretical expression for $\tau_{\text {late }}$, the equation is derived using Ferry's definition of $M_{e}$ (Ferry 1980), i.e., $M_{e}=\frac{\rho R T}{G_{N}^{0}}$, which should also be applied in their calculation. Instead, they use the value of $\mathrm{M}_{\mathrm{e}}$ reported by Fetters et al. (1994), which are $4 / 5$ as large as that defined by Ferry (1980) (e.g., $M_{e}$ of 1815 for 1,4 polybutadiene) $)^{1}$.

Double reptation model (Tsenoglou 1987, 1991; des Cloizeaux 1998)

The normalized relaxation modulus of the double reptation model is given simply by

\footnotetext{
${ }^{1}$ Please note the typo in the value of $G_{N}^{0}$ for 1,4 polybutadiene in Table 1 of Pattamaprom et al. (2000). The correct value of $G_{N}^{0}$ for 1,4 polybutadiene as reported in Fetters et al. (1994) is $1.25 \times 10^{6}$ $\mathrm{Pa}$.
}

$m_{d}(t)=\sum_{A} \sum_{B} w_{A}(t) w_{B}(t) p_{A}(t) p_{B}(t)=\left[\sum_{i} w_{i}(t) p_{i}(t)\right]^{2}$

where $\mathrm{m}_{\mathrm{d}}(\mathrm{t})$ is the average survival probability of entanglements at time $t, \mathrm{w}_{\mathrm{i}}$ is volume fraction of chain $i$. $\mathrm{p}_{\mathrm{i}}(\mathrm{t})$ is the tube survival probability of chain $i$ at time $t$, which in the form of double reptation considered here is calculated from the original theory of Doi and Edwards $(1978,1979)$ with a single relaxation time by

$p_{i}(t)=\sum_{k=\text { odd }}^{\infty} \frac{1}{k^{2}} \exp \left[-k^{2} t / \tau_{\text {rep }, i}\right] \sim \exp \left[-t / \tau_{\text {rep }, i}\right]$

where $\tau_{\text {rep,i }}$, the longest relaxation time of chain $i$, is obtained from an empirical dependence on molecular weight:

$$
\begin{aligned}
\tau_{\text {rep }, i} & =K^{\prime}\left(\frac{M_{w, i}}{M_{e}}\right)^{3.4} \\
& =K M_{w, i}^{3.4}
\end{aligned}
$$

Here $K$, an empirical parameter, can be set equal to $\frac{K^{\prime}}{M_{e}^{3.4}}$. The stress-relaxation modulus $\mathrm{G}_{\text {total }}(\mathrm{t})$ is directly proportional to $\mathrm{m}_{\mathrm{d}}(\mathrm{t})$ :

$G_{\text {total }}(t)=G_{N}^{0} m_{d}(t)=G_{N}^{0}\left[\sum_{i} w_{i}(t) p_{i}(t)\right]^{2}$

For monodisperse polymers, $\mathrm{G}_{\text {total }}(\mathrm{t})$ simplifies to $\mathrm{G}_{\text {total }}(\mathrm{t})=G_{N}^{0} \exp \left[\frac{-t}{\left(\tau_{\text {rep }, i} / 2\right)}\right]$, and $\eta_{0}$, the zero-shear viscosity, is simply related to $\tau_{\text {rep }}$ by

$\frac{\eta_{0}}{G_{N}^{0}}=\frac{\tau_{\text {rep }}}{2}=\frac{K}{2} M_{w}^{3.4}$

The parameters for the double reptation model are the plateau modulus $G_{N}^{0}$, and the empirical parameter $K$.

The stress-relaxation modulus $\mathrm{G}_{\text {total }}(\mathrm{t})$ from either model can then be converted to the storage modulus $\left(\mathrm{G}^{\prime}\right)$ and the loss modulus $\left(\mathrm{G}^{\prime \prime}\right)$ by

$$
\begin{aligned}
& G^{\prime}(\omega)=\omega \int_{0}^{\infty} G_{\text {total }}(t) \sin (\omega t) \mathrm{dt} \quad \text { and } \\
& G^{\prime \prime}(\omega)=\omega \int_{0}^{\infty} G_{\text {total }}(t) \cos (\omega t) \mathrm{d} t
\end{aligned}
$$

\section{Experimental}

Two monodisperse polystyrene melts were tested in our labs, one with the weight-average molecular weight $\left(\mathrm{M}_{\mathrm{w}}\right)$ of $4.11 \times 10^{5}\left(\mathrm{M}_{\mathrm{w}}\right.$ $M_{n}=1.06$ ) obtained from Pressure Chemical, the other with 
Table 1 List of experimental properties of monodisperse polystyrenes from several literature sources and the model parameters $\mathrm{K}$, $\tau_{\mathrm{e}}$, and $\mathrm{K}$ calculated from $\tau_{\mathrm{e}}\left(\mathrm{K}_{\mathrm{cal}}\right)$

\begin{tabular}{|c|c|c|c|c|c|c|}
\hline Literature & Mw & $\mathrm{Nen}^{\mathrm{a}}$ & $\begin{array}{l}\text { Temp. of } \\
\text { experiment }\left({ }^{\circ} \mathrm{C}\right)\end{array}$ & $\tau_{\mathrm{e}(\mathrm{s})}$ & $\mathrm{K}(\mathrm{s})$ & $\mathrm{K}_{\mathrm{cal}}^{\mathrm{b}}(\mathrm{s})$ \\
\hline \multirow[t]{3}{*}{ Schausberger et al. (1985) } & $289.97 \mathrm{~K}$ & 17.44 & $180 \mathrm{C}$ & 0.0013 & $5 \times 10^{-19}$ & $5.915 \times 10^{-19}$ \\
\hline & $750 \mathrm{~K}$ & 45.11 & $180 \mathrm{C}$ & 0.00098 & $5 \times 10^{-19}$ & $4.459 \times 10^{-19}$ \\
\hline & $3000 \mathrm{~K}$ & 180.45 & $180 \mathrm{C}$ & 0.00045 & $2.5 \times 10^{-19}$ & $2.048 \times 10^{-19}$ \\
\hline \multirow[t]{2}{*}{ Graessley and Roovers (1979) } & $275 \mathrm{~K}$ & 16.5 & $169.5 \mathrm{C}$ & 0.00196 & $8.8 \times 10^{-19}$ & $8.918 \times 10^{-19}$ \\
\hline & $860 \mathrm{~K}$ & 51.7 & $169.5 \mathrm{C}$ & 0.00196 & $8.8 \times 10^{-19}$ & $8.918 \times 10^{-19}$ \\
\hline \multirow{2}{*}{ Montfort et al. (1979) } & $160 \mathrm{~K}$ & 9.62 & $160 \mathrm{C}$ & 0.01 & $4.6 \times 10^{-18}$ & $4.55 \times 10^{-18}$ \\
\hline & $670 \mathrm{~K}$ & 40.3 & $160 \mathrm{C}$ & 0.01 & $4.6 \times 10^{-18}$ & $4.55 \times 10^{-18}$ \\
\hline Our Laboratory & $363 \mathrm{~K}$ & 21.83 & $150 \mathrm{C}$ & 0.05 & $2.25 \times 10^{-17}$ & $2.275 \times 10^{-17}$ \\
\hline
\end{tabular}

${ }^{\mathrm{a}} \mathrm{Using} \mathrm{M}_{\mathrm{e}}=16,625$ for polystyrene (Fetters et al. 1994) -where $M_{e}=\rho R T / G_{N}^{0}$ (Ferry 1980)

${ }^{\mathrm{b}} \mathrm{K}_{\mathrm{cal}}$ is calculated from the mapping between the double reptation model and the dual constraint model

$M_{w}=3.63 \times 10^{5}\left(M_{w} / M_{n}=1.03\right)$ from Polymer Source Inc. The GPC analyses of the $M_{w} / M_{n}$ were obtained from the manufacturers.

The dynamic oscillatory measurements of the polymer samples were measured from an Ares rheometer, a strain-controlled rheometer equipped with a force-rebalanced transducer from Rheometric Scientific Inc. We used cone and plate fixtures with a cone angle of 0.1 radians and a radius of $1.25 \mathrm{~cm}$. The frequency range of the measurements was from 0.001 to $100 \mathrm{~s}^{-1}$, and the measurements were done at $160{ }^{\circ} \mathrm{C}$ for the polystyrene with $\mathrm{M}_{\mathrm{w}}=4.11 \times 10^{5}$ and at $150^{\circ} \mathrm{C}$ for the polystyrene with $\mathrm{M}_{\mathrm{w}}=3.63 \times 10^{5}$, under nitrogen atmosphere to prevent degradation of the polymers.

\section{Results and discussion}

The discussion is divided into two parts: the first examines the validity of the dual constraint model and the double reptation model for thermorheologically simple polymers, and the second extends the dual constraint model predictions to linear and star-branched polyethylenes.

Test of the dual constraint model and the double reptation model for thermorheologically simple linear polymers

Monodisperse polymers: mapping of the dual-constraint model onto the double reptation model

For monodisperse linear polymers, both of the models, as expected, work well in the terminal regime, the regime where the zero-shear viscosity $\eta_{0}$ can be found. In addition, over a wide range of molecular weights, the dual constraint model predicts a dependence of $\eta_{0}$ on $\mathrm{M}_{\mathrm{w}}$ that approximates the 3.4 power law that is assumed by the double reptation model. Therefore, in the terminal regime the two models can be used interchangeably and their parameters can be found and correlated in this regime. We can therefore develop a mapping scheme to retrieve the model parameter $\mathrm{K}$ of the double reptation model from $\tau_{\mathrm{e}}$ of the dualconstraint model by matching the normalized zero-shear viscosity $\left(\eta^{*}\right)$ as a function of molecular weight for the two models.

In Fig. 1, the normalized viscosity, $\eta_{d c}^{*}=\frac{\eta_{0}}{G_{N}^{0} \tau_{e}}$, vs $\mathbf{M}_{\mathrm{w}}$ from the dual constraint model is superposed onto $\eta_{d r}^{*}\left(M_{w}\right)$ from the double reptation model, which is $\eta_{d r}^{*}=\frac{\eta_{0}}{G_{N}^{0} \tau_{e}}=\frac{K^{\prime}}{2 \tau_{e}}\left(\frac{M_{w}}{M_{e}}\right)^{3.4}=C_{1}\left(\frac{M_{w}}{M_{e}}\right)^{3.4}$

The value of $C_{1}=\frac{K^{\prime}}{2 \tau_{e}}=\frac{K M_{e}^{3.4}}{2 \tau_{e}}$ obtained from the superposition of $\eta_{d c}^{*}$ and $\eta_{d r}^{*}$ equals 0.051 , and this value should be independent of the chemical composition of the polymer and of the temperature. Thus, the general relationship between $K$ and $\tau_{\mathrm{e}}$ becomes $K=0.102 \frac{\tau_{e}}{M_{e}^{3.4}}$. Since $\tau_{\mathrm{e}}$ can either be obtained from the literature (if the monomeric friction coefficient is available) or from fitting the predictions of the dual-constraint model to experimental data, the parameter $K$ for each specific



Fig. 1 Mapping of the predictions of the dual constraint model (dash line with symbols) onto those of the double reptation model (solid line) using the relationship $\frac{\eta_{0}}{G_{N}^{0} \tau_{e}}=\frac{K^{\prime}}{2 \tau_{e}}\left(\frac{M_{w}}{M_{e}}\right)^{3.4}=C_{1}\left(\frac{M_{w}}{M_{e}}\right)^{3.4}$. The value of $\mathrm{C}_{1}$ obtained from the superposition equals 0.051 , independent of temperature or chemical composition of the polymer 
polymer can then easily be calculated. We test our method of mapping the dual constraint model parameter $\tau_{\mathrm{e}}$ onto the parameter $\mathrm{K}$ in the double reptation model using eight monodisperse polystyrenes listed in Table 1. As can be seen, the predicted $\mathrm{K}\left(\mathrm{K}_{\mathrm{cal}}\right)$ from the mapping procedure (col. 7) agrees well with the actual best-fit K obtained directly from double reptation model (col. 6). We note that for the data of Schausberger et al. (1985), the best-fit value of $\tau_{\mathrm{e}}$ at $180{ }^{\circ} \mathrm{C}$ varies with molecular weight. In our earlier paper (Pattamaprom et al. 2000), we noted that for a constant value of $\tau_{\mathrm{e}}$, the dual constraint model frequently underpredicts somewhat the moduli at low molecular weights (less than 20 entanglements per molecule) and over predicts them at large molecular weights (over 150 entanglements per molecule). Thus, a best-fit value of $\tau_{\mathrm{e}}$ varies somewhat if the molecular-weight range is very broad.

\section{Polydisperse polymers: rheological predictions of the dual-constraint and double reptation models}

Although the terminal-zone predictions from the two models are the same for monodisperse polymers, their

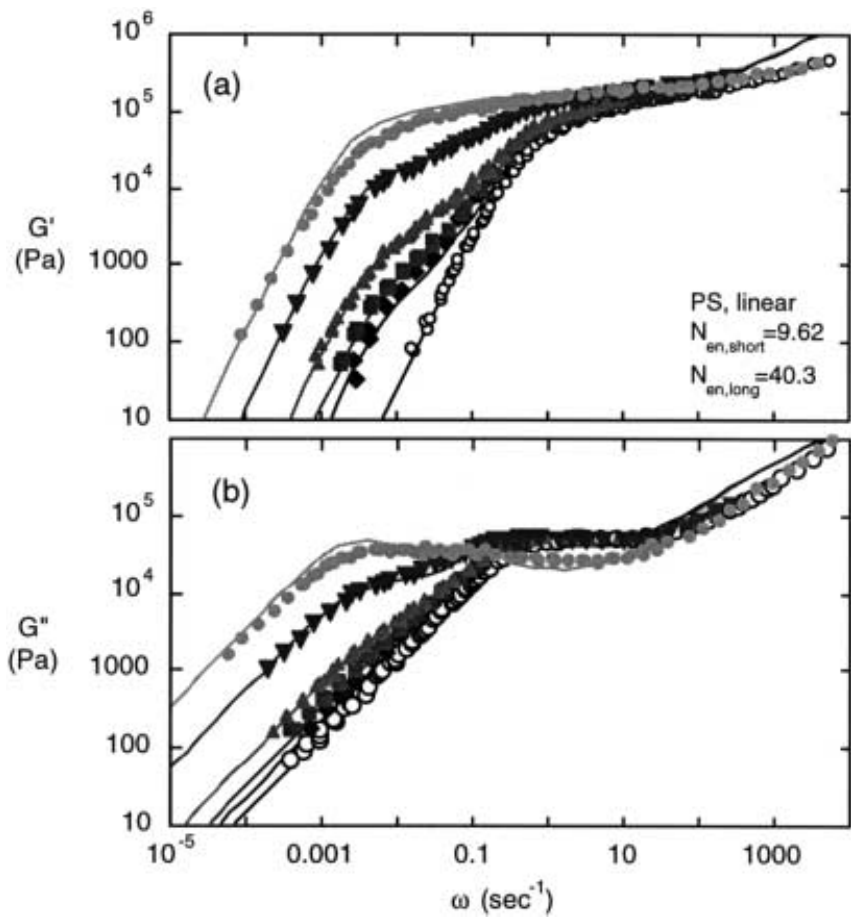

Fig. 2a, b Comparison of the dual-constraint-model predictions (solid lines) of: a storage modulus, $G^{\prime}$; b loss modulus, $G^{\prime \prime}$, with experimental data for linear binary blends of polystyrenes $\left(\mathrm{M}_{\mathrm{w}}=1.6 \times 10^{5}\right.$ and $\left.6.7 \times 10^{5}\right)$ at $160^{\circ} \mathrm{C}$ (Montfort et al. 1979), The volume fractions of the high-molecular-weight component $\left(\mathrm{x}_{1}\right)$ from right to left are $0.0,0.05,0.1,0.2,0.5$ and 1.0 , respectively. The model parameters are $G_{N}^{0}=2 \times 10^{5} \mathrm{~Pa}$ (Fetters et al. 1994) and $\tau_{\mathrm{e}}=0.01 \mathrm{~s}$ predictions differ in the cases of bidisperse and polydisperse polymers.

Bidisperse polymers. A comparison of the models with a set of bidisperse polystyrene experimental data at $160{ }^{\circ} \mathrm{C}$ from Montfort et al. (1979) is shown in Figs. 2 and 3 for a mixture of linear polystyrenes (PS) with molecular weights $1.6 \times 10^{5}$ and $6.7 \times 10^{5}$ at volume fractions $\mathrm{x}_{1}=0.05,0.1,0.2$, and 0.5 . The model parameters are $K$ (for the double reptation model $)=4.6 \times 10^{-18} \mathrm{~s}$ and $\tau_{\mathrm{e}}$ (for the dual constraint model) $=0.01 \mathrm{~s}$. These were taken from the best fits of the model predictions with the experimental data for monodisperse PS. As can be seen in Fig. 2, the dual-constraint model captures the experimental data well for both the monodisperse polystyrenes and their bidisperse mixtures. On the other hand, the predictions of the double reptation model are reasonably good only in the terminal regime. The inaccuracy of the double reptation model in the highfrequency regime is due to the omission of early-time fluctuations, dynamic tube dilution, and Rouse motions. Polydisperse polymers. For polymers with continuously distributed polydispersity, the shapes of the $G^{\prime}$ and $G^{\prime \prime}$ curves predicted by both models in the linear viscoelastic regime qualitatively resemble those of the experimental data at low frequencies. Quantitatively, however, there are still some discrepancies in the predictions from both models when using the same model parameters as in

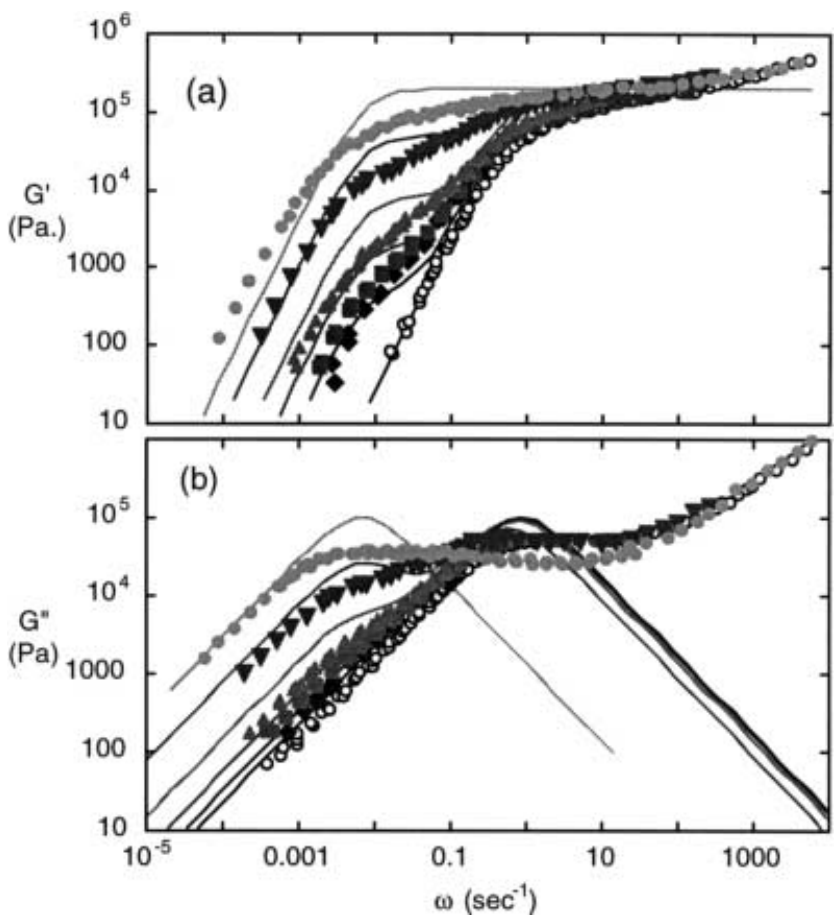

Fig. 3a, b Comparison of the double-reptation-model predictions (solid lines) of: a storage modulus, $\mathrm{G}^{\prime}$; b loss modulus, $\mathrm{G}^{\prime \prime}$, with the same experimental data as in Fig. 2 using the model parameter $G_{N}^{0}$ $2 \times 10^{5} \mathrm{~Pa}$ (Fetters et al. 1994) and $\mathrm{K}=4.6 \times 10^{-18} \mathrm{~s}$ 


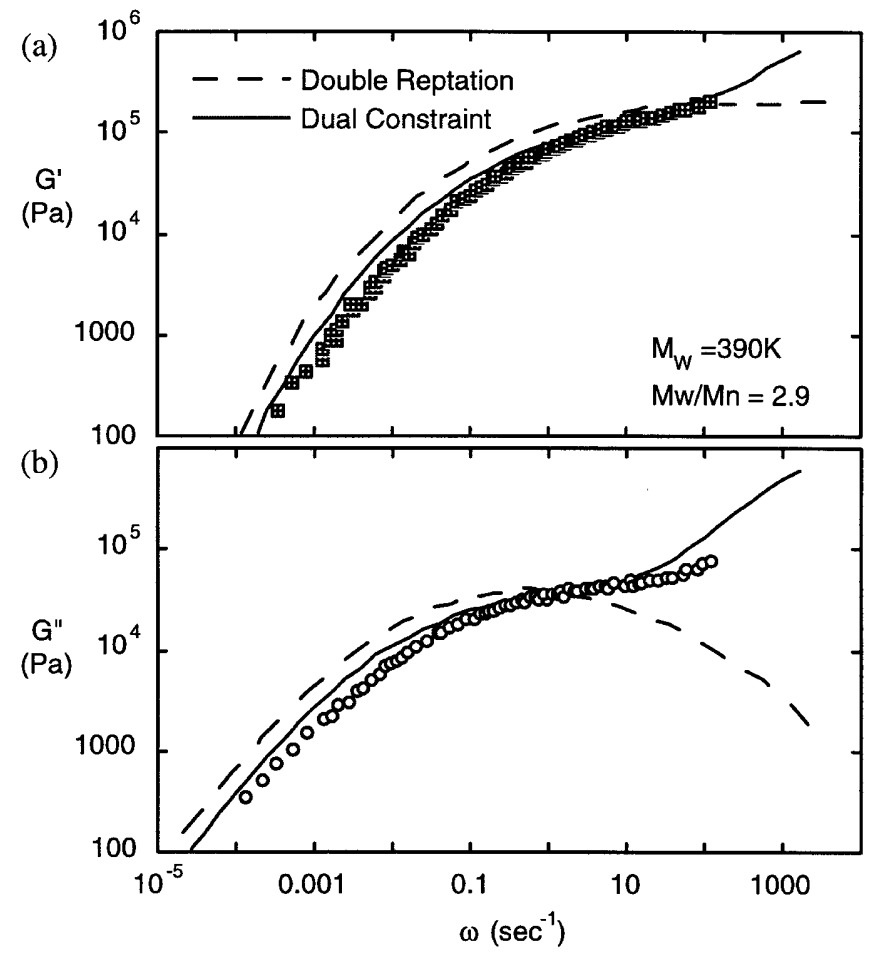

Fig. 4a, b Comparison of the dual constraint and the double reptation model predictions of: a storage modulus, $\mathrm{G}^{\prime}$; b loss modulus, $G^{\prime \prime}$, with experimental data for polydisperse polystyrene, $\mathrm{M}_{\mathrm{w}}=3.9 \times 10^{5}$ and $\mathrm{M}_{\mathrm{w}} / \mathrm{M}_{\mathrm{n}}=2.9$ at $160^{\circ} \mathrm{C}$. (Montfort et al. 1979) The solid lines are the dual-constraint-model predictions and the broken lines the double-reptation-model predictions using the same model parameters as in Figs. 2 and 3, respectively

monodisperse case. Figure 4 compares the predictions of $\mathrm{G}^{\prime}$ and $\mathrm{G}^{\prime \prime}$ at $160{ }^{\circ} \mathrm{C}$ by the two models to experimental data from Montfort et al. (1979) for polydisperse PS (PS8) with $\mathrm{M}_{\mathrm{w}}=3.9 \times 10^{5}$ and $\mathrm{M}_{\mathrm{w}} / \mathrm{M}_{\mathrm{n}}=2.9$ from GPC. The polydispersity was included in the model calculations by discretizing the GPC curve found in Montfort et al. (1979) into 20 discrete components. As can be seen, the model comparison using the same time constant as for the monodisperse and bidisperse PS, i.e., $\tau_{\mathrm{e}}=0.01 \mathrm{~s}$ and $\mathrm{K}=4.6 \times 10^{-18} \mathrm{~s}$, predicts the shape of the experimental data well, but there is a shift in position of the predicted curves relative to the experimental ones. Quantitatively, however, the prediction of the dual constraint model is more accurate than that of the double reptation model. In order to investigate the terminal-regime predictions more thoroughly, we will next focus on the zero-shear viscosity predicted from the models and compare it with experimental data from the literature.

The values of zero-shear viscosity as a function of molecular weight and molecular weight distribution for both the double reptation model and the dual-constraint model are shown in Fig. 5. The distribution considered here is a log-normal distribution. As can be seen, when


Fig. 5a, b The predictions of the normalized zero-shear viscosity vs the average number of entanglements per chain $\left(\mathrm{M}_{\mathrm{w}} / \mathrm{M}_{\mathrm{e}}\right)$ from: a the dual constraint model; $\mathbf{b}$ the double reptation model

$\mathrm{M}_{\mathrm{w}} / \mathrm{M}_{\mathrm{n}}<2$, the predictions from both the doublereptation model and the dual-constraint model are insensitive to polydispersity; hence, we expect the mapping depicted in Fig. 1 to still be valid in this regime. However, when $\mathrm{M}_{\mathrm{w}} / \mathrm{M}_{\mathrm{n}}>2$, the predictions from the two models are different and the mapping procedure is invalid. The double reptation model shows significantly higher zero-shear viscosities for higher polydispersity, while the predictions from the dual constraint model show the opposite, though much weaker, trend. This prediction of the double reptation model was also recently shown by Tuminello (2000). The difference between the predictions of the two models contributes mainly from the tube dilation mechanism. Without tube dilation, the predictions of the dual constraint model becomes similar to those of the double reptation model, as can be seen from Fig. 6.

Next, we test the predictions of the models by comparing their predictions to experimental data for monodisperse and polydisperse samples from several literature sources (listed in Table 2). Some of the data at $170{ }^{\circ} \mathrm{C}$ were shifted to the reference temperature of $160{ }^{\circ} \mathrm{C}$ using WLF shift factor from Schausberger et al. (1985). (We avoid shifting the data from temperatures lower than $160{ }^{\circ} \mathrm{C}$, since $\eta_{0}$ is extremely temperature sensitive at low temperature.) The $\eta_{0}$ values from the 


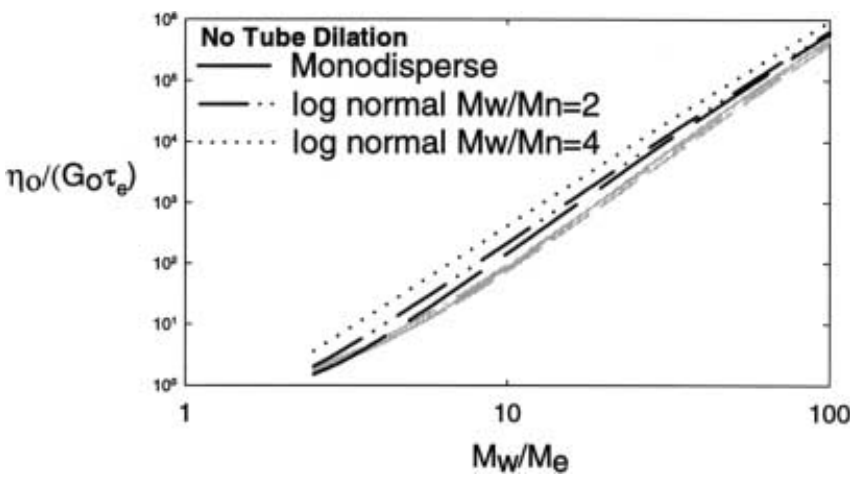

Fig. 6 Comparison of the normalized zero-shear viscosity vs the average number of entanglements per chain $\left(\mathrm{M}_{\mathrm{w}} / \mathrm{M}_{\mathrm{e}}\right)$ using the dual constraint model with tube dilation (gray lines) and without tube dilation (black lines). The lines in grey-scale are the prediction of the model with tube dilation reproduced from Fig. 5a

experiments were compared against the predictions of the dual constraint model in Fig. 7a and against the double reptation model in Fig. $7 \mathrm{~b}$. The distribution considered here is again a log-normal distribution. In order to identify the polydisperse polystyrene data points that were obtained from the WLF shifting, we use open symbols $(\bigcirc)$, while closed symbol $(\bullet)$ are used for polydisperse samples at $160{ }^{\circ} \mathrm{C}$ with no shifting. As expected, both models predict $\eta_{0}$ for monodisperse PS reasonably well. The $\eta_{0}$ data of most polydisperse PS samples appear to be slightly lower than those for the monodisperse counterparts, which agrees with the predictions of the dual constraint model. Nevertheless, the scatter in the data and the lack of clear correlation between $\mathbf{M}_{\mathrm{w}} / \mathrm{M}_{\mathrm{n}}$ and the magnitude of the decrease in $\eta_{\mathrm{o}}$ leaves open the possibility that $\eta_{0}$ might be independent of $M_{w} / M_{n}$ at fixed $M_{w}$. This finding at least partially supports the prediction of the dual-constraint model and disagrees with the double reptation model. However, there is ambiguity in this result due to possible imperfections in the GPC analysis of the polydispersity of the samples and the fact that the dual-constraint model may be inaccurate at low molecular weight, i.e., when the number of entanglements per chain is less than ten.

To eliminate ambiguity due to the GPC analysis, the experimental data at $150{ }^{\circ} \mathrm{C}$ obtained from a polydisperse polystyrene $\left(\mathrm{M}_{\mathrm{w}, \mathrm{avg}}=357 \mathrm{~K}, \mathrm{M}_{\mathrm{w}} / \mathrm{M}_{\mathrm{n}}=2.3\right)$ prepared from a mixture of 11 monodisperse components by Wassermann and Graessley (1992) is investigated together with experimental data from a monodisperse PS having similar weight-average molecular weight $\left(\mathrm{M}_{\mathrm{w}}=363 \mathrm{~K}, \mathrm{M}_{\mathrm{w}} / \mathrm{M}_{\mathrm{n}}=1.03\right)$ tested in our laboratory (see Experimental section). By fitting the dual constraint model to the experimental data for the monodisperse PS at $150{ }^{\circ} \mathrm{C}$, we obtain a good fit using $\tau_{\mathrm{e}}=0.05 \mathrm{~s}$; the model parameter $K$ of the double reptation model obtained from the mapping correlation mentioned earlier, i.e., $K=0.102 \frac{\tau_{e}}{M_{.}^{3.4}}$, is $\mathrm{K}=2.27 \times 10^{-17}$ s Comparisons of the predictions of these models to the
Table 2 Summary of the experimental monodisperse, bidisperse, and polydisperse polystyrenes from several literature sources used in Fig. 7

\begin{tabular}{|c|c|c|c|c|c|}
\hline Literature & $\mathrm{M}_{\mathrm{w}}$ & $\mathrm{Nen}^{\mathrm{a}}$ & $\mathrm{M}_{\mathrm{w}} / \mathrm{M}_{\mathrm{n}}$ & $\begin{array}{l}\text { Temp. of } \\
\text { Experiment } \\
\left({ }^{\circ} \mathrm{C}\right)\end{array}$ & $\begin{array}{l}\eta_{0, \text { ref at }} \text { at } \\
160^{\circ} \mathrm{C}(\mathrm{Pa}-\mathrm{s})\end{array}$ \\
\hline \multicolumn{6}{|l|}{ a) "Monodisperse" PS } \\
\hline \multirow[t]{7}{*}{ Montfort et al. (1984) } & $35 \mathrm{~K}$ & 2.11 & 1.06 & 160 & $3.02 \times 10^{3}$ \\
\hline & $100 \mathrm{~K}$ & 6.0 & 1.06 & 160 & $5.75 \times 10^{4}$ \\
\hline & $110 \mathrm{~K}$ & 6.62 & 1.05 & 160 & $7.94 \times 10^{4}$ \\
\hline & $200 \mathrm{~K}$ & 12.03 & 1.06 & 160 & $6.03 \times 10^{5}$ \\
\hline & $390 \mathrm{~K}$ & 23.46 & 1.10 & 160 & $4.36 \times 10^{6}$ \\
\hline & $900 \mathrm{~K}$ & 54.14 & 1.12 & 160 & $6.92 \times 10^{6}$ \\
\hline & $2700 \mathrm{~K}$ & 162.4 & 1.20 & 160 & $1.59 \times 10^{7}$ \\
\hline \multirow[t]{2}{*}{ Montfort et al. (1979) } & $160 \mathrm{~K}$ & 9.62 & $\mathrm{na}^{\mathrm{ii}}$ & 160 & $1.35 \times 10^{5}$ \\
\hline & $670 \mathrm{~K}$ & 40.3 & $\mathrm{na}^{\mathrm{ii}}$ & 160 & $3.18 \times 10^{7}$ \\
\hline Our Laboratory & $411 \mathrm{~K}$ & 24.72 & 1.06 & 160 & $4.82 \times 10^{6}$ \\
\hline \multicolumn{6}{|l|}{ b) Polydisperse PS } \\
\hline \multirow[t]{2}{*}{ Münstedt (1980) } & $219 \mathrm{~K}$ & 13.17 & 2.3 & 160 & $3.7 \times 10^{5}$ \\
\hline & $253 \mathrm{~K}$ & 15.22 & 1.9 & 160 & $8.6 \times 10^{5}$ \\
\hline Leonardi et al. (2000) & $326 \mathrm{~K}$ & 19.6 & 3.4 & 160 & $2.67 \times 10^{6}$ \\
\hline Montfort et al. (1979) & $390 \mathrm{~K}$ & 23.46 & 2.9 & 160 & $2.3 \times 10^{6}$ \\
\hline Montfort et al. (1986) & $227 \mathrm{~K}$ & 13.65 & 2.17 & 160 & $5.0 \times 10^{5}$ \\
\hline Laun (1984) & $398 \mathrm{~K}$ & 23.94 & 2.9 & 170 & $2.93 \times 10^{6^{*}}$ \\
\hline \multirow[t]{2}{*}{ Laun and Schuch (1989) } & $205.3 \mathrm{~K}$ & 12.35 & 1.28 & 170 & $7.52 \times 10^{5^{*}}$ \\
\hline & $1306.7 \mathrm{~K}$ & 78.60 & 2.49 & 170 & $2.31 \times 10^{8^{*}}$ \\
\hline
\end{tabular}

${ }^{\mathrm{a}} \mathrm{Using} \mathrm{M}_{\mathrm{e}}=16,625$ for polystyrene (Fetters et al. 1994) -where $M_{e}=\rho R T / G_{N}^{0}$ (Ferry 1980)

ii na - a nearly monodisperse polymer with no $\mathrm{M}_{\mathrm{w}} / \mathrm{M}_{\mathrm{n}}$ reported in the literature

$* \eta_{0, \text { ref }}$ is shifted from the reported temperature of $170{ }^{\circ} \mathrm{C}$ to $160{ }^{\circ} \mathrm{C}$ using WLF parameters from Schausberger et al. (1985) 


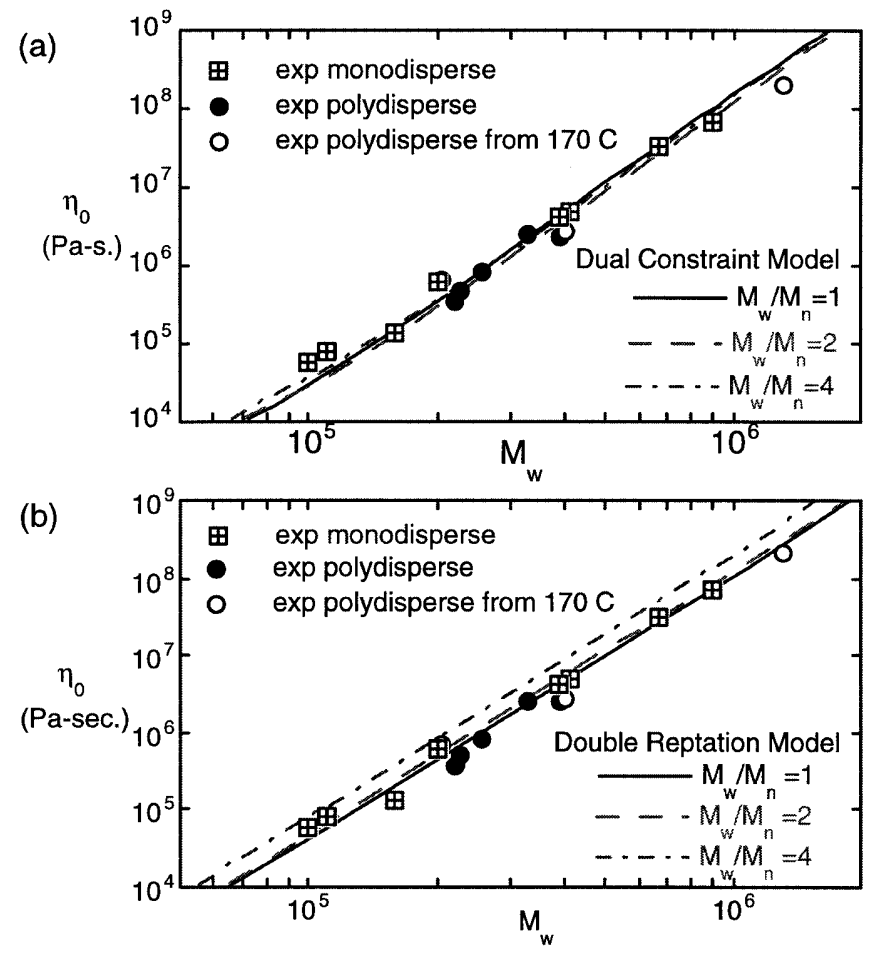

Fig. 7a, b Comparison of $\eta_{0}$ predicted from: a the dual constraint model; $\mathbf{b}$ the double reptation model, with the experimental data for monodisperse (plus sign in square) and polydisperse (filled circle) polystyrenes reported in Table 2 at a reference temperature $\mathrm{T}_{\mathrm{r}}=160{ }^{\circ} \mathrm{C}$. The data points with (open circle) indicate the polydisperse polystyrene data that were shifted from $170{ }^{\circ} \mathrm{C}$. The model parameters for the dual constraint and the double reptation models are the same as those in Figs. 2 and 3, respectively

experimental data are shown in Fig. 8a. The same model parameters (i.e., $\tau_{\mathrm{e}}=0.05 \mathrm{~s}, \mathrm{~K}=2.27 \times 10^{-17} \mathrm{~s}$ ) are then used to predict the polydisperse PS experimental data of Wassermann and Graessley. (Fig. 8b) As can be seen, the prediction of the dual constraint model agrees well with the experimental data, while the double reptation model slightly overestimates the terminal relaxation time (implying that $\eta_{0}$ is predicted to be too high). Since the dual constraint model predicts a slight decrease to no change in viscosity as the polydispersity increases, and the double reptation model predicts an increase in viscosity with increasing polydispersity, this result supports the notion that the zero-shear viscosity is nearly independent of polydispersity.

We believe that the failure of the double reptation model to predict quantitatively the zero-shear viscosity of polydisperse polymers is due to the lack of tube dilation as a mechanism of constraint release. As can be seen in Fig. 6, once the dynamic dilution mechanism is removed from the dual constraint model, the prediction shows an increase in $\eta_{0}$ as the polydispersity increases, similar to that predicted by double reptation. Nevertheless, for bidisperse polymers with a small amount of long
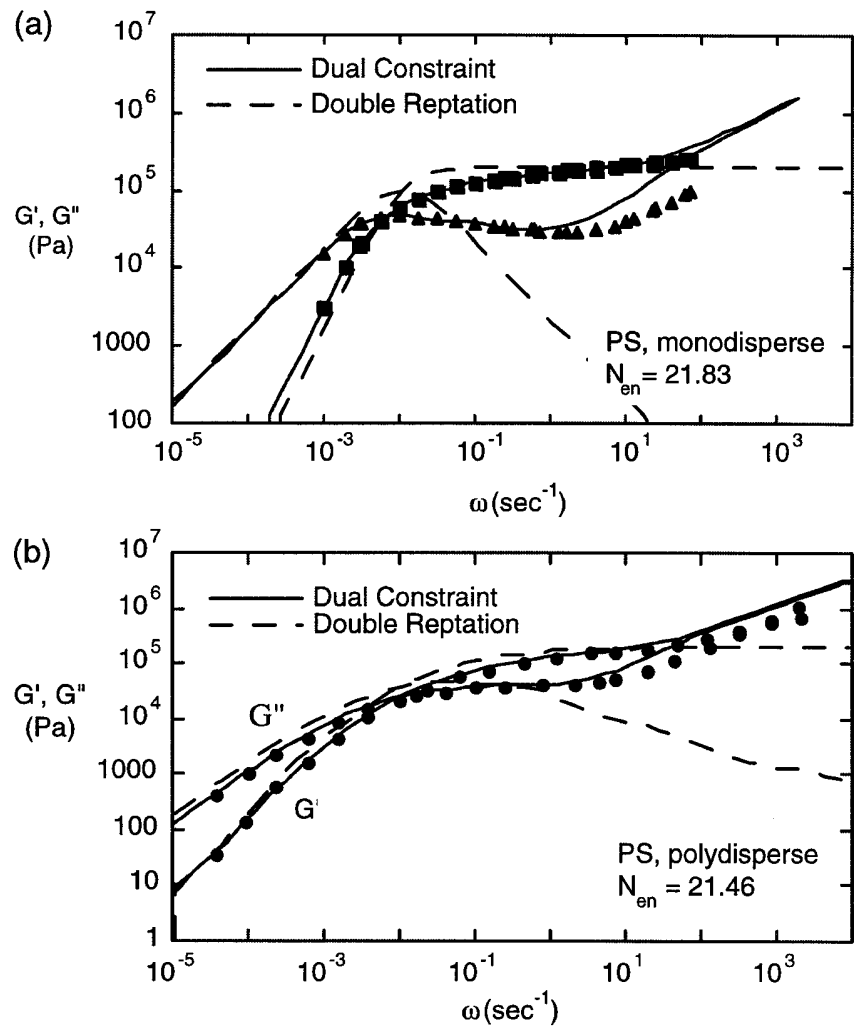

Fig. 8a, b Comparison of the dual constraint (solid lines) and the double reptation (broken lines) model predictions of storage modulus $\left(\mathrm{G}^{\prime}\right)$ and loss modulus $\left(\mathrm{G}^{\prime \prime}\right)$ with experimental data for: a a monodisperse polystyrene $\left(\mathrm{M}_{\mathrm{w}}=363 \mathrm{~K}\right)$; b a polydisperse polystyrene $\left(\mathrm{M}_{\mathrm{w}, \text { avg }}=357 \mathrm{~K}, \mathrm{M}_{\mathrm{w}} / \mathrm{M}_{\mathrm{n}}=2.3\right)$ (Wasserman and Graessley 1992) at $\mathrm{T}_{\mathrm{r}}=150{ }^{\circ} \mathrm{C}$. The model parameters are $G_{N}^{0}=2 \times 10^{5} \mathrm{~Pa}$. (Fetters et al. 1994), $\tau_{\mathrm{e}}=0.05 \mathrm{~s}$, and $\mathrm{K}=2.275 \times 10^{-17} \mathrm{~s}$

polymer chains in matrix of short chains, the failure of the double reptation model is also attributed to the lack of another constraint release mechanism called constraint release Rouse relaxation.

Investigation of hydrogenated polybutadienes and polyethylenes

In the previous section and in Pattamaprom et al. (2000), we tested the dual constraint theory with polystyrenes, polybutadienes, and polyisoprenes. The dual constraint model captures the rheological behavior of these polymers well for both the linear and star architectures. In this section we will discuss the validity of the dual constraint theory for linear and star polyethylenes, the latter of which are notorious as thermorheologically complex polymers. Described below are investigations of model monodisperse linear polyethylenes with $2 \%$ ethyl branches, namely hydrogenated polybutadienes (h-PBD), polydisperse 
commercial polyethylenes (PE), and model starbranched h-PBDs.

\section{Monodisperse linear $h-P B D s$}

For linear h-PBD, we present comparisons of the predictions of the dual constraint theory with the $\mathrm{G}^{\prime}$ and $\mathrm{G}^{\prime \prime}$ experimental data and with the zero-shear viscosities $\eta_{0}$. In general, the dual constraint model predictions agree well with the experimental data as also seen with the other polymers investigated.

Figure 9 shows that the $G^{\prime \prime}$ experimental data for linear h-PBD at $190{ }^{\circ} \mathrm{C}$ from Raju et al. (1979) agree well with the dual constraint model predictions for the whole range of molecular weight studied, using the bestfit model parameter $\tau_{\mathrm{e}}=7 \times 10^{-9} \mathrm{~s}$, and the literature value $G_{N}^{0}=2.31 \times 10^{6} \mathrm{~Pa}$. (Raju et al. 1979).

In Fig. 10, the experimental zero-shear viscosities $\eta_{0}$ for linear h-PBD from several sources (Pearson et al. 1994; Tao et al. 2000; Raju et al. 1979) are compared with the predicted values from the dual constraint model at the reference temperature of $190{ }^{\circ} \mathrm{C}$ using the aforementioned model parameters, i.e., $\tau_{\mathrm{e}}=7 \times 10^{-9} \mathrm{~s}$, $G_{N}^{0}=2.31 \times 10^{6} \mathrm{~Pa}$. The experimental data from Tao et al. (2000) and Pearson et al. (1994) were shifted to $190{ }^{\circ} \mathrm{C}$ using the average value of the Arrhenius correlation proposed by Arnett and Thomas (1980) shown below:

$\left.\ln \eta_{0, h-P B D}=\ln M^{3.41}+\frac{(3523)}{T} \exp [(2.36) \times b)\right]-37.04$

where $\mathrm{M}$ is the molecular weight of a linear polyethylene, $\mathrm{T}$ is the absolute temperature $(\mathrm{K})$, and $\mathrm{b}$ is the fraction of ethyl branches in a polymer chain. The h-PBD considered here contains $2 \%$ ethyl branches,

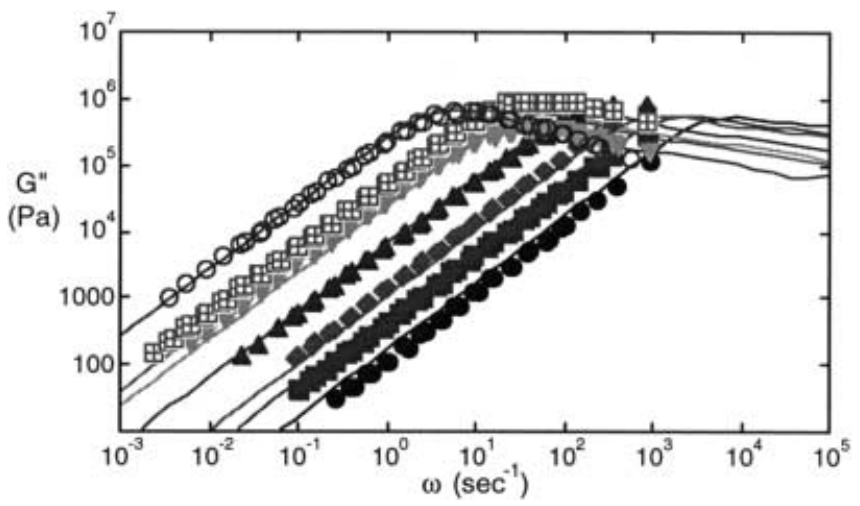

Fig. 9 Comparison of the prediction of the dual constraint model (lines) with $\mathrm{G}^{\prime \prime}$ experimental data for linear h-PBD at $190{ }^{\circ} \mathrm{C}$ (Fig. 2 of Raju et al. 1979) using the model parameters $G_{N}^{0}=2.31 \times 10^{6} \mathrm{~Pa}$. and $\tau_{\mathrm{e}}=7 \times 10^{-9} \mathrm{~s}$ thus giving b equal to 0.02 . Also shown in Fig. 10 is the empirical correlation between $\eta_{0}$ and $\mathrm{M}$ for linear h-PBD (with $2 \%$ ethyl branches) at $190{ }^{\circ} \mathrm{C}$ from Eq. (18) (-..-). As can be seen, the prediction of the dual constraint model agrees well with the experimental data and to within $20 \%$ with the correlation for h-PBD from Arnett and Thomas.

\section{Polydisperse linear PEs}

In this part, we progress toward the predictions of polydisperse commercial linear polyethylenes. We compare the predictions of the dual constraint model with experimental data for several polyethylenes at $190{ }^{\circ} \mathrm{C}$ from different literature sources including a metallocenecatalyzed linear high-density polyethylene (HDPE) (Rohlfing and Janzen 1999), conventional linear HDPEs (Wood-Adams and Dealy 1996), and branched PEs (Shroff and Mavridis 1999). The polydispersity of these polyethylenes was included in the model calculations by discretizing the GPC curves reported in the corresponding literature into 20 discrete components. The model parameters for high-density polyethylene were taken from Fetters et al. (1994) for $G_{N}^{0}$, i.e., $G_{N}^{0}=2.6 \times 10^{6} \mathrm{~Pa}$ and $M_{e}=\frac{G_{N}^{0}}{\rho k_{B} T}=1035 . \tau_{\mathrm{e}}$ was obtained by matching $\eta_{0}$ from Arnett's correlation (Eq. 18) with that of the dualconstraint model prediction for polyethylene, leading to $\tau_{\mathrm{e}}=7 \times 10^{-9} \mathrm{~s}$ at $190{ }^{\circ} \mathrm{C}$ (same value as $\tau_{\mathrm{e}}$ for h-PBD). For linear low-density polyethylene (LLDPE), data fitting shows that $\tau_{\mathrm{e}}$ is slightly larger, $\tau_{\mathrm{e}}=11 \times 10^{-9} \mathrm{~s}$, than for HDPE, as might be expected, since LLDPE contains short side branches, while HDPE is nominally free of side branches.

The dual-constraint-model prediction of the linear metallocene-catalyzed HDPE is compared with the experimental data reported by Rohlfing and Janzen

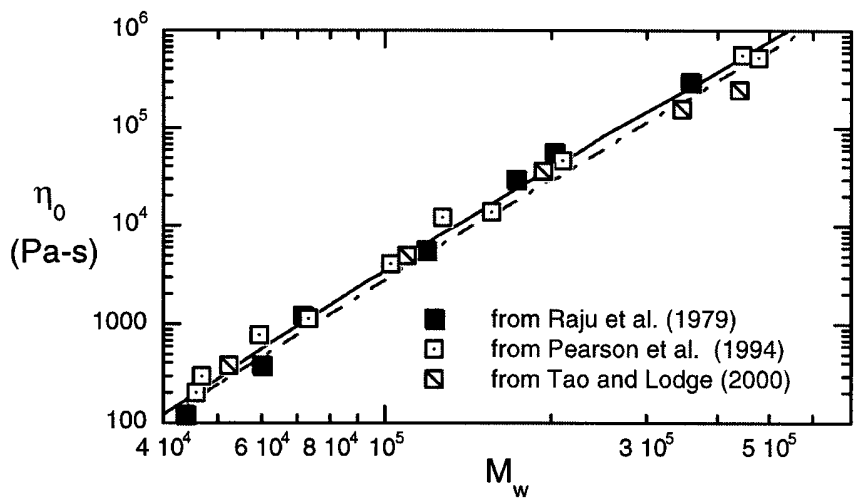

Fig. 10 Comparison of $\eta_{0}$ predicted from the dual constraint model (solid line) with $\eta_{0}$ data obtained experimentally for monodisperse linear h-PBD at $\mathrm{T}_{\mathrm{r}}=190{ }^{\circ} \mathrm{C}$ from several literature sources. Also shown is the correlation proposed by Arnett and Thomas (1980) (---) 
(1999) for HDPE with $\mathrm{M}_{w}=98,840, \mathrm{M}_{\mathrm{w}} / \mathrm{M}_{\mathrm{n}}=3.57$ (from GPC) at $190{ }^{\circ} \mathrm{C}$ in Fig. 11. As can be seen, the prediction agrees well with the linear metallocene HDPE (O). Also shown are data for the branched HDPE ( $\square$ ) produced by treating the linear HDPE with peroxide. The comparison in Fig. 11 illustrates that the effect of long-chain branching is dominant in the low-frequency regime. The same indication is also observed from the comparison of the dual constraint model predictions with the experimental data for linear and branched LLDPEs at $190{ }^{\circ} \mathrm{C}$ (Gabriel and Münstedt 2000) (Fig. 12). These experimental data cover seven decades of frequencies, and were able to reach the terminal regime, even for the branched sample. These two polymers have almost the same molecular weight distribution, as indicated by the GPC curves in the insert to Fig. 12a. As can be seen, the prediction for the linear LLDPE can be fit quite well to the experimental data (Fig. 12a) by choosing $\tau_{\mathrm{e}}=11 \times 10^{-9} \mathrm{~s}$, while the prediction of the theory for linear polymers with this same value of $\tau_{\mathrm{e}}$ deviates from the data for branched LLDPE at low frequencies (Fig. 12b), as is also the case for the branched HDPE in Fig. 11.

Figure 13 shows a comparison of the dual-constraint model prediction with the experimental data of the conventional HDPE synthesized using Ziegler-Natta catalyst with unknown amount of antioxidant $\left(\mathrm{M}_{\mathrm{w}}=4.11 \times 10^{4}, \mathrm{M}_{\mathrm{w}} / \mathrm{M}_{\mathrm{n}}=2.9\right.$, and $\mathrm{M}_{\mathrm{w}}=3.19 \times 10^{5}$, $\mathrm{M}_{\mathrm{w}} / \mathrm{M}_{\mathrm{n}}=31$ ) at $190{ }^{\circ} \mathrm{C}$ (Wood-Adams and Dealy 1996). The model parameters are the same as in Fig. 11. As can be seen, in Fig. 13 the predictions agree qualitatively with the experimental data; however, the magnitudes of the experimental $\eta^{*}$ value are lower than the predicted values. We suspect that the presence of antioxidant and

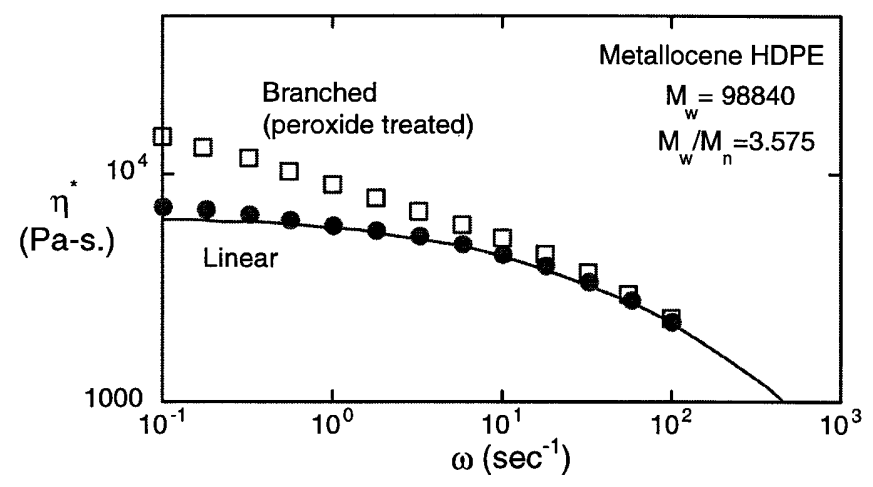

Fig. 11 Comparison of the dual-constraint-model predictions (line) of the frequency-dependent dynamic viscosity $\eta^{*}$ with experimental data (filled circle) for metallocene-catalyzed linear $\operatorname{HDPE}\left(\mathrm{M}_{\mathrm{w}}=9.88 \times 10^{4}\right.$ with $\mathrm{M}_{\mathrm{w}} / \mathrm{M}_{\mathrm{n}}=3.6$ ) at $190{ }^{\circ} \mathrm{C}$. The model parameters are $G_{N}^{0}=2.6 \times 10^{6} \mathrm{~Pa}$ (Fetters et al. 1994) and $\tau_{\mathrm{e}}=7 \times 10^{-9} \mathrm{~s}$. The $\eta^{*}$ for branched HDPE (open square) obtained from treating the linear HDPE precursor with peroxide is also shown for comparison. The experimental data are from Rohlfing and Janzen (1999) degree of short-chain branching might play roles in decreasing the plateau modulus.

Finally, we compare the dual-constraint model predictions with two more polymers that contain longchain branches in Fig. 14. Although we expect the dualconstraint model to fail in this case, the discrepancy between the predicted and the experimental viscosities will provide us with information on the effect of branching. The values of $\eta^{*}$ for linear polymers with small degree of long-chain branching were reported in Bersted et al. (1981) to be higher than those of the linear polymers at low frequencies, and lower at high frequencies. The branched polymers investigated here consist of a metallocene branched PE (Dow1140) with $\mathrm{M}_{\mathrm{w}}=8.5 \times 10^{4}, \mathrm{M}_{\mathrm{w}} / \mathrm{M}_{\mathrm{n}}=2.4$, and long chain branching index LCBI $=0.51$, and a branched LLDPE (Dow2045) with $\mathrm{M}_{\mathrm{w}}=13.8 \times 10^{4}, \mathrm{M}_{\mathrm{w}} / \mathrm{M}_{\mathrm{n}}=4.2$, and $\mathrm{LCBI}=0.13$ (Shroff and Mavridis 1999). The comparisons between the dual-constraint model predictions and these experimental data agree with those reported in Bersted et al. in that at low frequencies, $\eta^{*}$, of both polymers is higher than predicted for linear polymers of the same molecular weight. Also the metallocene polymer with $\mathrm{LCBI}=0.51$ shows not only enhanced viscosity at low frequencies but also a reduced viscosity at high frequencies, relative to the prediction for linear polymers of the same molecular weight distribution, which is also similar to the observation of Bersted et al.

\section{Star-branched h-PBDs}

For star-branched h-PBD, we find anomalous behavior when comparing the experimental data from the literature with the predictions of the dual constraint model. As described in detail below, we find that the star h-PBD has a lower plateau modulus $G_{N}^{o}$ than that of the linear counterparts, and that the effective energy of fluctuations $\left(\mathrm{U}_{\mathrm{eff}}\right)$ for the 3-arm and the 4-arm stars are different.

The experimental $\eta_{\mathrm{o}}$ data were taken from Table II of Graessley and Raju (1984). These data are compared with the predictions from the dual constraint model for star polymers shown in Fig. 15. As can be seen in Fig. $15 \mathrm{a}$, the 3 -arm stars have lower values of $\eta_{0}$ than that of the 4-arm stars at the same arm molecular weight $\mathbf{M}_{\mathrm{w} \text {,arm }}$; the viscosity differences are as high as a decade at high $\mathbf{M}_{\mathrm{w}, \mathrm{arm}}$. Also, although both 3-arm and 4-arm h-PBD stars seem to have an exponential dependence of $\eta_{0}$ on $\mathbf{M}_{\mathrm{w}, \mathrm{arm}}$, the steepness of the exponential dependence of $\eta_{0}$ seems to be lower for the 3-arm than for the 4-arm stars. This implies that the effective energy of fluctuations $\left(U_{\text {eff }}\right)$ incorporated in the exponential term of the dual constraint model (Eqs. 2 and 4) for the 3- and 4-arm stars are different. Although a lower viscosity for the 3-arm than for 
4-arm stars has also been found in several other polymers (e.g., polyisoprene stars) (Fetters et al. 1993; Klein 1986), the decrease in viscosity is not as dramatic as it is for h-PBD. Strangely, the $\eta_{0}$ values for star h-PBDs seem to correlate better with the total molecular weight $\mathbf{M}_{\mathrm{w} \text {,total }}$ than with the arm molecular weight $\mathbf{M}_{\mathrm{w}, \text { arm }}$, as shown in Fig. 15b. The correlation with the limited data shows a power law of 6.4 for star h-PBD compared to the power 3.4 for linear h-PBD. This phenomenon is at odds with simple polymers like polyisoprenes, where $\eta_{0}$ of the 3-arm stars were reported to be only $20 \%$ lower than that for higher numbers of arms at the same arm molecular weight (Fetters et al. 1993), and the dependence of $\eta_{0}$ on $\mathbf{M}_{\mathrm{w} \text {,arm }}$ follows the same exponential power for any number of arms, as shown in Fig. 16. The anomalous behavior of the star-branched h-PBD was also addressed in Levine and Milner (1998). Their findings agree with ours that the molecular weight between entanglements for star polymers is effectively bigger than that of the linear polymers, and the plateau modulus for star polymers needs to be adjusted to obtain good fits with experimental data. Nevertheless, the difference between the molecular weight between entanglements for 3-arm and 4-arm star h-PBD was not reported in their paper.

For star h-PBD, $\mathrm{U}_{\text {eff }}$ defined in Eq. (4) can be adjusted by changing $M_{e}$ from that of the linear h-PBD (i.e., $M_{e}=1250$ ) to account for the difference in the effective energy of fluctuations for the 3 -arm and the 4-arm stars from that of the linear counterparts. Hereafter, the adjusted $\mathrm{M}_{\mathrm{e}}$ that provides the best fit to the $\eta_{0}-\mathrm{M}_{\mathrm{w}, \text { arm }}$ relationships will be called $\mathrm{M}_{\mathrm{e} \text {,eff. As }}$ shown in Fig. 15a, good agreement between the dual constraint model predictions and the experimental data can be achieved when $\mathbf{M}_{e, \text { eff }}$ is 3000 for the 3-arm
Fig. 12a, b Comparison of the dual-constraint-model predictions (line) with $\mathrm{G}^{\prime}, \mathrm{G}^{\prime \prime}$, and $\eta^{*}$ experimental data for; a linear LLDPE (mLLDPE4, $\mathrm{M}_{\mathrm{w}}=$ $\left.1.14 \times 10^{5}, \mathrm{M}_{\mathrm{w}} / \mathrm{M}_{\mathrm{n}}=2.07\right) ; \mathbf{b}$ branched LLDPE (mLLDPE3, $\mathrm{M}_{\mathrm{w}}=9.86 \times 10^{4}, \mathrm{M}_{\mathrm{w}} / \mathrm{M}_{\mathrm{n}}=$ 2.05) at $190{ }^{\circ} \mathrm{C}$. The model parametersare $G_{N}^{0}=2.6 \times 10^{6} \mathrm{~Pa}$ (Fetters et al. 1994) and the value $\tau_{\mathrm{e}}=11 \times 10^{-9} \mathrm{~s}$, which is a best fit to the data of $\mathbf{a}$. The GPC traces for both data are also shown in the small figure in Fig. 12a. The experimental data are from Gabriel and Münstedt (2000). Note that the value of the best-fit $\tau_{\mathrm{e}}$ in this case is larger than in Fig. 11. This is potentially due to the lower $G_{N}^{0}$ for the LLDPEs than for HDPEs
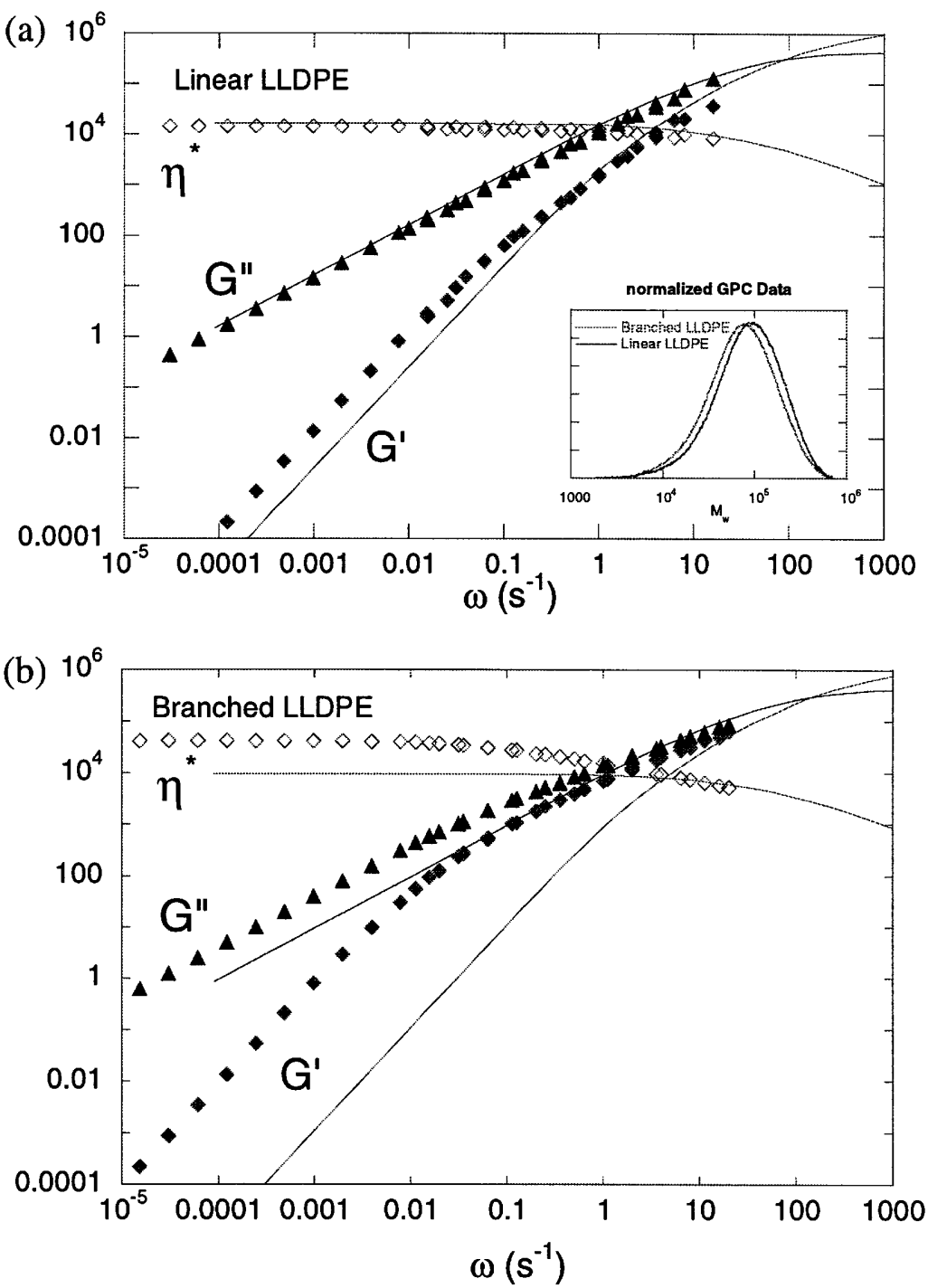


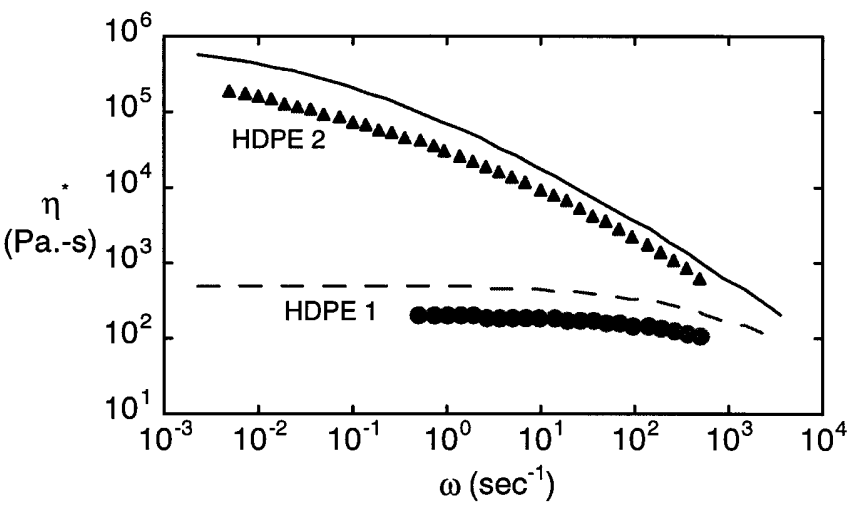

Fig. 13 Comparisons of the dual-constraint-model predictions of dynamic viscosity $\eta^{*}$ with experimental data for two linear HDPEs treated with antioxidant; HDPE1 $\left(\mathrm{M}_{\mathrm{w}}=4.11 \times 10^{4}\right.$ with $\mathrm{M}_{\mathrm{w}} / \mathrm{M}_{\mathrm{n}}=$ 2.9) and HDPE $2\left(\mathrm{M}_{\mathrm{w}}=3.19 \times 10^{5}\right.$ with $\left.\mathrm{M}_{\mathrm{w}} / \mathrm{M}_{\mathrm{n}}=31\right)$ at $190{ }^{\circ} \mathrm{C}$. (Wood-Adams and Dealy 1996). The model parameters are the same as in Fig. 11. The broken line and the solid line are the predictions for linear HDPE having the same $\mathrm{M}_{\mathrm{w}}$ and $\mathrm{M}_{\mathrm{w}} / \mathrm{M}_{\mathrm{n}}$ as for HDPE1 and HDPE2, respectively

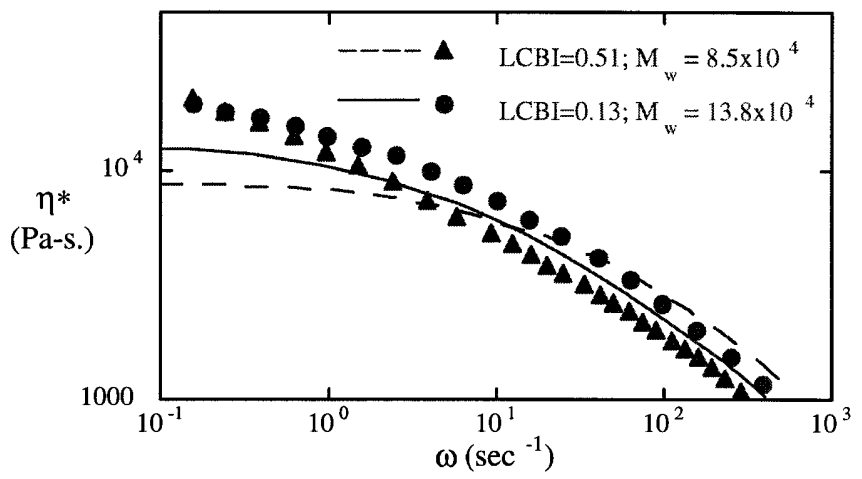

Fig. 14 Comparison of the dual-constraint-model predictions of dynamic viscosity $\eta^{*}$ with experimental data for a metallocenecatalyzed branched PE $\left(\mathrm{M}_{\mathrm{w}}=8.5 \times 10^{4}\right.$ with $\mathrm{M}_{\mathrm{w}} / \mathrm{M}_{\mathrm{n}}=2.4, \mathrm{LCBI}=$ $0.51)$ (filled triangle) and LLDPE $\left(\mathrm{M}_{\mathrm{w}}=13.8 \times 10^{4}\right.$ with $\mathrm{M}_{\mathrm{w}} /$ $\mathrm{M}_{\mathrm{n}}=4.2, \mathrm{LCBI}=0.13$ ) (filled circle) at $190{ }^{\circ} \mathrm{C}$, where LCBI is a long-chain branching index indicating the amount of long chain branches defined in Eq. (11) of Shroff and Mavridis (1999). The model parameters are the same as in Fig. 12 for LLDPE. The broken and solid lines are the predictions for linear PE having the same $\mathrm{M}_{\mathrm{w}}$ and $\mathrm{M}_{\mathrm{w}} / \mathrm{M}_{\mathrm{n}}$ as the metallocene-catalyzed branched PE and the LLDPE, respectively

stars, and 2000 for the 4-arm stars. Note that the higher $\mathbf{M}_{\mathrm{e}, \mathrm{eff}}$ implies lower exponential power, and thus lower $\mathrm{U}_{\mathrm{eff}}$.

A comparison of the dual constraint predictions with the $G^{\prime}$ and $G^{\prime \prime}$ experimental data for star h-PBDs (Raju et al. 1979) shows that after adjusting $U_{\text {eff }}$ correspondingly for the 3- and the 4-arm stars as mentioned above, the predictions agree with the experimental data only when the plateau modulus is set to $5 \times 10^{5} \mathrm{~Pa}, 4.6$ times smaller than that of the linear counterpart. (see Fig. 17) The reduced $G_{N}^{o}$ and the difference in $\mathrm{U}_{\text {eff }}$ found in
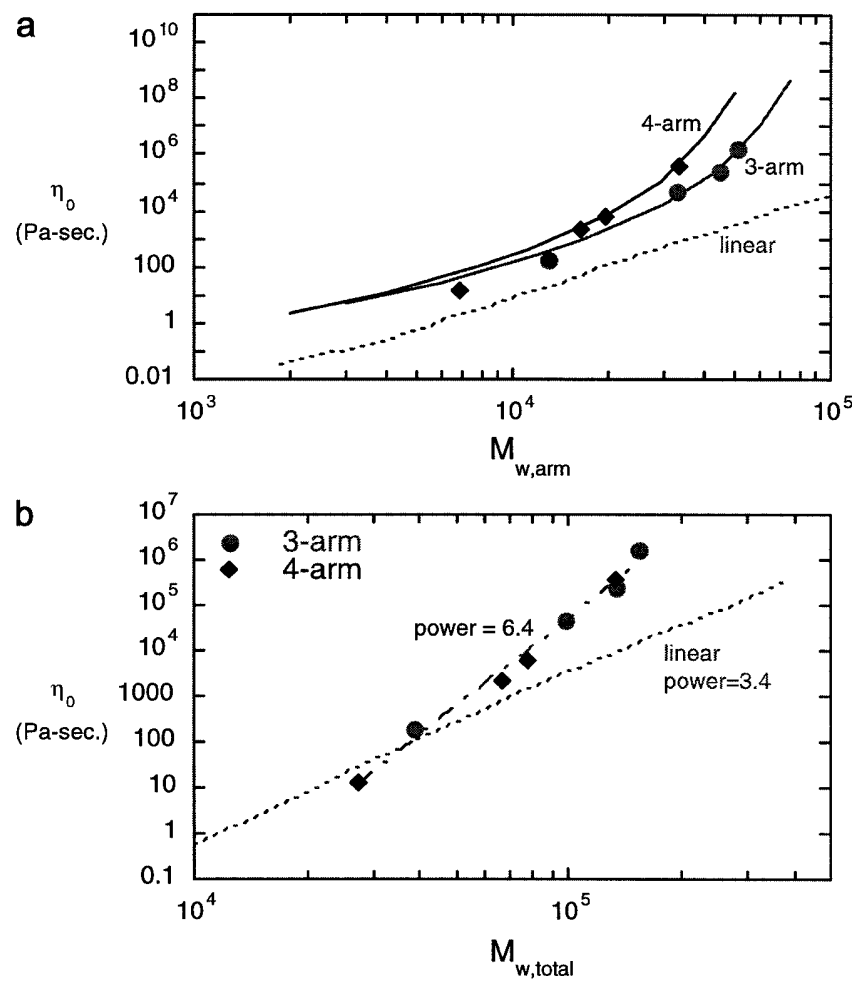

Fig. 15 a Experimental $\eta_{0}$ for 3-arm (filled circle) and 4-arm (filled diamond) star h-PBDs at $190{ }^{\circ} \mathrm{C}$ (Graessley and Raju 1984) plotted as a function of $\mathrm{M}_{\mathrm{w}, \text { arm }}$ compared with the predictions from the dual constraint model for the 3-arm (-) and the 4-arm (-) stars, respectively, using $G_{N}^{0}=5.0 \times 10^{5} \mathrm{~Pa}$. For the 3-arm stars, $\mathrm{M}_{\mathrm{e}, \mathrm{eff}}=$ 3000 , and $\tau_{\mathrm{e}}=1.4 \times 10^{-5} \mathrm{~s}$, and, for the 4-arm stars, $\mathrm{M}_{\mathrm{e}, \mathrm{eff}}=2000$, and $\tau_{\mathrm{e}}=6 \times 10^{-6} \mathrm{~s}$. b The same experimental data as in a plotted as a function of $\mathrm{M}_{\mathrm{w}, \text { total }}$ agrees well with the 6.4-power correlation (-*-). Also shown is the $\eta_{0}$ for linear h-PBD at the same temperature (……)

h-PBD are anomalous in that these adjustments are not required for other sets of polymers previously investigated (i.e., polystyrene, polybutadiene, and polyisoprene) (Pattamaprom et al. 2000). The decrease in the activation energy of fluctuations of star h-PBD is an indication of lower entropic activation barrier for fluctuations in branched polyethylenes compared to other branched polymers. We are inclined to conclude that these anomalies as well as the thermorheological complexity observed for branched polyethylenes (Graessley 1982; Carella et al. 1986) are related to the contour-length-fluctuation mechanisms specific to polyethylene with long-chain branches. However, the availability of experimental data for star h-PBD is too limited to reach a definite conclusion.

\section{Summary and future directions}

Since for linear monodisperse polymers the linear viscoelastic rheological predictions of the double repta- 


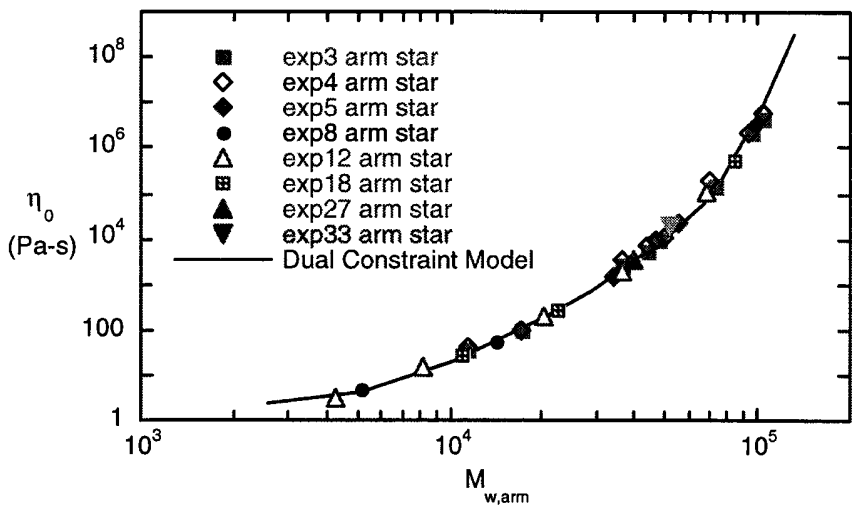

Fig. 16 Comparison of $\eta_{0}$ predicted from the dual constraint model with experimental data for monodisperse star-branched polyisoprenes at a reference temperature of $60{ }^{\circ} \mathrm{C}$ from Fetters et al. (1993). Model parameters: $G_{N}^{0}=4.34 \times 10^{5} \mathrm{~Pa}$. (Pearson et al. 1983) and $\tau_{\mathrm{e}}=1.2 \times 10^{-5} \mathrm{~s}$



Fig. 17 Comparison of $G^{\prime \prime}$ predictions from the dual constraint model (-) with experimental data for star h-PBD at $190{ }^{\circ} \mathrm{C}$ using the same model parameters as in Fig. 15a. Also shown is the best-fit predictions using the same $G_{N}^{0}$ as for linear h-PBD, i.e., $G_{N}^{0}=2.31 \times 10^{6} \mathrm{~Pa}$., $\tau_{\mathrm{e}}=1.3 \times 10^{-6} \mathrm{~s}(\ldots \ldots \ldots)$

tion and the dual constraint models are equally accurate in the terminal regime, we have developed a mapping procedure whereby the parameter $\mathrm{K}$ of the double reptation theory can be obtained from the Rouse relaxation time of an entanglement segment $\tau_{\mathrm{e}}$ of the dual constraint model as $K=0.102 \frac{\tau_{e}}{M^{3.4 .}}$. In turn, $\tau_{\mathrm{e}}$ can be obtained from the monomeric friction factor $\zeta$ by $\tau_{e}=\frac{\zeta a^{2} M_{e}}{3 \pi^{2} k_{B} T M_{0}}$ where $a$ is the tube diameter, $\mathrm{M}_{\mathrm{e}}$ the entanglement spacing, and $\mathrm{M}_{0}$ the monomer molecular weight. This mapping procedure was found to work well when the polydispersity ratio $\mathrm{M}_{\mathrm{w}} / \mathrm{M}_{\mathrm{n}}$ is less than 2 . For bidisperse polymers, with parameters fixed using monodisperse data, the dual constraint model is more accurate than the double reptation model even in the terminal regime. For polydisperse polymers with the molecular weight distributions obtained from GPC, the dual constraint model provides better quantitative agreement with the experimental data than does the double reptation model using the same model parameters as were found for monodisperse polymers. For polydisperse linear PS and PE, the dual constraint model provides good agreement with the experimental data, while the double reptation model is somewhat inaccurate. The majority of experimental zero-shear viscosities for the polydisperse PS samples appear to have the same, or slightly lower, zero-shear viscosities than monodisperse polystyrenes of equal weight-averaged molecular weight. This result agrees with the prediction of the dual constraint model, while the double reptation model predicts the opposite trend. The success of the dual constraint model in predicting accurately $\eta^{*}$ for linear polydisperse polyethylenes allows us to distinguish the effect of polydispersity from that of long-chain branching.

From our investigation on monodisperse h-PBDs, which are the model monodisperse polyethylenes, we find that linear monodisperse h-PBD follows the 3.4 power law dependence of $\eta_{0}$ on molecular weight and the dual-constraint model can predict the linear viscoelastic properties of these polymers well. On the other hand, for star-branched h-PBD, we need to adjust separately the plateau modulus and $\mathbf{M}_{\mathrm{e}, \mathrm{eff}}$ for the 3-arm and 4-arm stars to get a good fit, which is thought to be related to the observed thermorheological complexity of branched PE. The increase in $\mathbf{M}_{\mathrm{e}, \mathrm{eff}}$ for star h-PBDs implies a lower entropic activation barrier for contour-length fluctuations in branched polyethylenes compared to other polymers.

Acknowledgement This research was supported by the NSF (DMR 9807262). CP is grateful for a Royal Thai Fellowship from the government of Thailand.

\section{Appendix: Comparison of dual constraint model of dynamic dilution with Ball-McLeish theory for stars}

Here we explore the relationship between the dual constraint "two-tube" model for dynamic dilution and the self-consistent theory of Ball and McLeish for monodisperse, highly entangled, star polymers. We begin by noting that the relaxation time of a piece of the star arm in a fixed, undiluted matrix is given by (Ball and McLeish 1989; Pattamaprom et al. 2000):

$\tau^{*}=\tau_{R} \exp \left\{v \mathrm{~N}_{\mathrm{en}}\left(1-\mathrm{s}^{*}\right)^{2}\right\}$

where $\mathrm{s}^{*}$ is the coordinate of the fixed tube, running from 0 at the branch point to unity at the branch tip, $\tau_{R}$ is the Rouse time for relaxation of the arm, $\mathrm{N}_{\mathrm{en}}$ is the number of undiluted entanglements of the arm, and $v=15 / 8$ is a constant. In the diluted tube, the relaxation time is assumed by the dual constraint model to be (Pattamaprom et al. 2000) 
$\tau=\tau_{R} \exp \left\{v \mathrm{~N}_{\mathrm{en}}(1-\mathrm{s})^{2} \phi^{*}(\mathrm{t})\right\}$

where $\mathrm{s}$ is the tube coordinate in the diluted tube and $\phi^{*}(\mathrm{t})$ is the fraction of unrelaxed arm in the undiluted tube. The function $\phi^{*}(\mathrm{t})$ is calculated from the relaxation of an arm in a fixed tube.

The Ball-McLeish theory assumes a clean separation of time scales - that at any time during relaxation the arm can be divided into two pieces, an outer piece that is completely relaxed, and an inner piece that is not at all relaxed. The tube survival probabilities $\mathrm{p}^{*}\left(\mathrm{~s}^{*}, \mathrm{t}\right)$ and $\mathrm{p}(\mathrm{s}, \mathrm{t})$ in the fixed and dilating tubes are then inwardtraveling waves whose shape is a Heaviside step function. Since the ratio of the relaxation time of the core of the star branch to that of the tip increases exponentially with $\mathrm{M}_{\mathrm{a}}$, the molecular weight of the arm, this approximation becomes progressively better as $\mathrm{M}_{\mathrm{a}}$ increases. Within this approximation, the "two-tube" model gives for the tube segment survival probability function $\mathrm{p}^{*}\left(\mathrm{~s}^{*}, \mathrm{t}\right)$ in the fixed tube:

$p^{*}\left(s^{*}, \mathrm{t}\right)=0$, for $\mathrm{s}_{\mathrm{c}}^{*}(\mathrm{t}) \geq \mathrm{s}^{*} \geq 0$

and

$\mathrm{p}^{*}\left(\mathrm{~s}^{*}, \mathrm{t}\right)=1, \quad$ for $0 \geq \mathrm{s}^{*} \geq \mathrm{s}_{\mathrm{c}}^{*}(\mathrm{t})$

where $s_{c} *(t)$ is the position along the fixed tube that separates the relaxed from the unrelaxed part of the arm. The dilution function $\phi^{*}(\mathrm{t})$, which is the fraction of unrelaxed arm in the fixed tube, is therefore given by $\phi^{*}(\mathrm{t})=\mathrm{s}_{\mathrm{c}}^{*}(\mathrm{t})$.

In the diluted tube, the tube segment survival probability $\mathrm{p}(\mathrm{s}, \mathrm{t})$ in the limit of high arm molecular weight is described by a similar Heaviside step function $\mathrm{p}(\mathrm{s}, \mathrm{t})=0, \quad$ for $\mathrm{s}_{\mathrm{c}}(\mathrm{t}) \geq \mathrm{s} \geq 1$

and

$\mathrm{p}(\mathrm{s}, \mathrm{t})=1, \quad$ for $0 \geq \mathrm{s} \geq \mathrm{s}_{\mathrm{c}}(\mathrm{t})$

We now compute the longest relaxation time $\tau_{\mathrm{f}}$ from our "two-tube" model in the high-molecular-weight limit. During relaxation, the dilution factor $\phi^{*}(\mathrm{t})$ decreases from unity to some as yet unknown value $\phi_{\mathrm{f}}^{*}=\phi^{*}\left(t_{\mathrm{f}}\right)$ at time $t_{f}$, where the time $t_{f}$ corresponds to complete relaxation. Because of the wide spectrum of time scales in star relaxation, one can take $t_{\mathrm{f}} \approx \tau_{\mathrm{f}}$.

The value of $\tau_{\mathrm{f}}$ is set by the condition that relaxation of the arm in the fixed tube must have progressed to the point that only the fraction $\phi_{\mathrm{f}}^{*}=\mathrm{s}_{\mathrm{c}}^{*}\left(\mathrm{t}_{\mathrm{f}}\right)$ of the arm remains unrelaxed. Since this occurs at time $t_{\mathrm{f}} \approx \tau_{\mathrm{f}}$, we have from Eq. (A1):

$\mathrm{t}_{\mathrm{f}}=\tau_{\mathrm{R}} \exp \left\{v \mathrm{~N}_{\mathrm{en}}\left[1-\mathrm{s}_{\mathrm{c}}^{*}\left(\mathrm{t}_{\mathrm{f}}\right)\right]^{2}\right\}=\tau_{\mathrm{R}} \exp \left\{v \mathrm{~N}_{\mathrm{en}}\left(1-\phi_{\mathrm{f}}^{*}\right)^{2}\right\}$

Now, the time $t_{f}$ corresponds to complete relaxation in the diluted matrix, so that $\mathrm{s}_{\mathrm{c}}\left(\mathrm{t}_{\mathrm{f}}\right)=0$. From Eq. (A2) we then have that the longest relaxation in the dilated tube is

$\mathrm{t}_{\mathrm{f}}=\tau_{\mathrm{R}} \exp \left\{v \mathrm{~N}_{\mathrm{en}} \phi_{\mathrm{f}}^{*}\right\}$

Equating Eqs. (A5) and (A6) gives

$\left(1-\phi_{\mathrm{f}}^{*}\right)^{2}=\phi_{\mathrm{f}}^{*}$

The solution to this quadratic equation is $\phi_{\mathrm{f}}^{*}=(3-\sqrt{5}) / 2=0.382$. Using this diluent arm fraction $\phi_{\mathrm{f}}^{*}$, the longest relaxation time can be calculated as $\tau_{\mathrm{f}}=\tau_{\mathrm{R}} \exp \left(0.382 v \mathrm{~N}_{\mathrm{en}}\right)$, which is close to the BallMcLeish result, $\tau_{R} \exp \left(v \mathrm{~N}_{\mathrm{en}} / 3\right)$. The diameter of the outer tube at the completion of relaxation can be calculated as $a=\frac{a_{0}}{\sqrt{\phi_{f}^{*}}}$. Thus, the outer-tube diameter grows modestly over the course of the fluctuationdriven relaxation process. This result provides a direct contrast with the Ball-McLeish description where the tube diameter becomes infinite when the arm has completely relaxed. Nevertheless, the similarity of the longest relaxation time of the dual constraint model with that of the Ball-McLeish theory shows that, for predicting the effect of dynamic dilution on stress, the dual constraint model is a good approximation to the Ball-McLeish theory.

\section{References}

Arnett RL, Thomas CP (1980) Zero-shear viscosity of some ethyl branched paraffinic model polymers. J Phys Chem 84:649-652

Ball RC, McLeish TCB (1989) Dynamic dilution and the viscosity of star polymer melts. Macromolecules 22:1911-1913

Bersted BH, Slek JD, Richter CA (1981) Prediction of rheological behavior of branched polyethylene from molecular structure. J Appl Polym Sci 26:10011014
Carella JM, Gotro JT, Graessley WW (1986) Thermorheological effects of long-chain branching in entangled polymer melts. Macromolecules 19: 659-667

des Cloizeaux J (1988) Double reptation vs. simple reptation in polymer melts. J Europhys Lett 5:437-442

Doi M, Edwards SF (1978) Dynamics of concentrated polymer systems, parts 13. J Chem Soc Faraday Trans II 74 : $1789-1832$
Doi M, Edwards SF (1979) Dynamics of concentrated polymer systems, part 4 rheological properties $\mathrm{J}$ Chem Soc Faraday Trans II 75:38-54

Doi M, Edwards SF (1986) The theory of polymer dynamics. Clarendon, Oxford

Ferry JD (1980) Viscoelastic properties of polymers. Wiley, New York

Fetters LJ, Kiss AD, Pearson DS (1993) Rheological behavior of star-shaped polymers. Macromolecules 26:647-654 
Fetters LJ, Lohse DJ, Richter D, Witten TA, Zirkel A (1994) Connection between polymer molecular weight, density, chain dimensions, and melt viscoelastic properties. Macromolecules 27:4639-4647

Gabriel C, Münstedt H (2000) Influence of long-chain branches on shear creep and recovery behaviour of polyethylene melts. XIIIth International Congress on Rheology, pp 1-231

Graessley WW (1982) Effect of long branches on the temperature-dependence of viscoelastic properties in polymer melts. Macromolecules 15:11641167

Graessley WW, Roovers J (1979) Melt rheology of four-arm and six-arm star polystyrenes. Macromolecules 12:959965

Graessley WW, Raju VR (1984) Some rheological properties of solutions and blends of hydrogenated polybutadiene. J Polym Sci Polym Symp 71:77-93

Janzen J, Colby RH (1999) Diagnosing long-chain branching in polyethylenes. J Mol Struct 486:569-584

Klein J (1986) Dynamics of entangled linear, branched, and cyclic polymers. Macromolecules 19:105-118

Laun HM (1984) Stresses and recoverable strains of stretched polymer melts and their prediction by means of a single integral constitutive equation. Proceedings of the 9th International Congress of Rheology, Acapulco, Mexico

Laun HM, Schuch (1989) Transient elongational viscosities and drawability of polymer melts. J Rheol 33:119-175

Léonardi F, Allal A, Marin G (1998) Determination of the molecular weight distribution of linear polymers by inversion of a blending law on complex viscosities. Rheol Acta 37:199-213

Léonardi F, Majesté JC, Allal A, Marin G (2000) Rheological models based on the double reptation mixing rule: the effects of a polydisperse environment. J Rheol 44:675-692

Levine AJ, Milner S (1998) Star polymers and the failure of time-temperature superposition. Macromolecules 31:86238637

Marrucci G (1985) Relaxation by reptation and tube enlargement: a model for polydisperse polymers. J Polym Sci Polym Phys Ed 23:159-177
Mead DW (1994) Determination of molecular weight distributions of linear flexible polymers from linear viscoelastic material functions. J Rheol 38:17971827

Mead DW, Van Dyke TJ, Larson RG, Doi M (1997) Private communication

Milner ST (1996) Relating the shear-thinning curve to the molecular weight distribution in linear polymer melts. Macromolecules 40:303-315

Milner ST, McLeish TCB (1997) Parameter-free theory for stress relaxation in star polymer melts. Macromolecules 30: $2159-2166$

Milner ST, McLeish TCB (1998) Reptation and contour-length fluctuations in melts of linear polymers. Phys Rev Lett 81:725-728

Milner ST, McLeish TCB, Young RN, Hakiki A, Johnson JM (1998) Dynamic dilution, constraint-release, and starlinear blends. Macromolecules 31:9345-9353

Montfort JP, Marin G, Arman J, Monge P (1979) Viscoelastic properties of high molecular weight polymers in the molten state I: Influence of the molecular weight distribution on linear viscoelastic properties. Rheol Acta 18:623-628

Montfort JP, Marin G, Monge P (1984) Effects of constraint release on the dynamics of entangled linear polymer melts. Macromolecules 17:1551

Montfort JP, Marin G, Monge P (1986) Molecular weight distribution dependence of the viscoelastic properties of linear polymers: the coupling of reptation and tube-renewal effects. Macromolecules 19:1979

Münstedt H (1980) Dependence of the elongation behavior of polystyrene melts on molecular weight and molecular weight distribution. J Rheol 24:847

Pattamaprom C, Larson RG, Van Dyke TJ (2000) Quantitative predictions of linear viscoelastic rheological properties of entangled polymers. Rheol Acta (submitted)

Pearson DS, Mueller SJ, Fetters LJ, Hadjichristidis N (1983) Comparison of the rheological properties of linear and star-branched polyisoprenes in shear and elongational flows. J Polym Sci Polym Phys Ed 21:2287-2298

Pearson DS, Fetters LJ, Graessley WW, Ver Strate G, von Meerwall E (1994) Viscosity and self-diffusion coefficient of hydrogenated polybutadiene. Macromolecules 27:711

Raju VR, Rachapudy H, Graessley WW (1979) Properties of amorphous and crystallizable hydrocarbon polymers. 4 Melt rheology of linear and starbranched hydrogenated polybutadiene. J Polym Sci Polym Phys Ed 17:1223

Rohlfing DC, Janzen J (1999) Melt-rheological characteristics of metallocenecatalyzed polyethylenes (book chapter). In: Scheirs J (ed) Metallocene-based polyolefins-preparation, properties and technology, vol 2. Wiley, Chichester, pp 419-434

Schausberger A, Schindlauer G, JaneschitzKriegl H (1985) Linear elastico-viscous properties of molten standard polystyrenes.: Presentation of complex moduli; role of short range structural parameters. Rheol Acta 24:220-227

Shroff RN, Mavridis H (1999) Long-chainbranching index for essentially linear polyethylenes. Macromolecules 32:8454-8464

Tao H, Lodge TP, von Meerwall ED (2000) Diffusivity and viscosity of concentrated hydrogenated polybutadiene solutions. Macromolecules 33:1747-1758

Tsenoglou C (1987) Viscoelasticity of binary homopolymer blends. ACS Polym Prepr 28:185-186

Tsenoglou C (1991) Molecular-weight polydispersity effects on the viscoelasticity of entangled linear-polymers. Macromolecules 24:1762-1767

Tuminello WH (1986) Molecular weight and molecular weight distribution from dynamic measurements of polymer melts. Polym Eng Sci 26:1339-1347

Tuminello WH (2000) Determination of molecular weight distributions from rheological properties of polymer melts. Rheol Bull 69:8-15

Viovy JL, Rubinstein M, Colby RH (1991) Constraint release in polymer melts: tube reorganization versus tube dilution. Macromolecules 24:3587-3596

Wasserman SH, Graessley WW (1992) Effects of polydispersity on linear viscoelasticity in entangled polymer melts. J Rheol 36:543-572

Wood-Adams PM, Dealy JM (1996) Use of rheological measurements to estimate the molecular weight distribution of linear polyethylene. J Rheol 40:761778 